\title{
Current concepts in neuroanatomical tracing
}

\author{
C. Köbbert ${ }^{\mathrm{a}}$, R. Apps ${ }^{\mathrm{b}}$, I. Bechmann ${ }^{\mathrm{c}}$, J.L. Lanciego ${ }^{\mathrm{d}}$, J. Mey ${ }^{\mathrm{e}}$, S. Thanos ${ }^{\mathrm{a}, *}$ \\ ${ }^{a}$ Department of Experimental Ophthalmology, Medical School, University of Müenster, Domagkstraße 15, D-48149 Muenster, Germany \\ ${ }^{\mathrm{b}}$ Motor Control Group, Department of Physiology, School of Medical Sciences, University of Bristol Bristol BS8 1TD, UK \\ ${ }^{\mathrm{c}}$ Department of Cell and Neurobiology, Institute of Anatomy, Humboldt University Hospital Charité, D-10098 Berlin, Germany \\ ${ }^{\mathrm{d}}$ Departamento de Anatomí, Facultad de Medicina, Universidad de Navarra, Irunlarrea s/n. E-31080 Pamplona, Spain \\ ${ }^{\mathrm{e}}$ Institute of Biology II, RWTH, Kopernikusstraße 16, D-52074 Aachen, Germany
}

Received 3 February 2000

\begin{abstract}
The development of new axonal tract tracing and cell labelling methods has revolutionised neurobiology in the last 30 years. The aim of this review is to consider some of the key methods of neuroanatomical tracing that are currently in use and have proved invaluable in charting the complex interconnections of the central nervous system. The review begins with a short overview of the most frequently used tracers, including enzymes, peptides, biocytin, latex beads, plant lectins and the everincreasing number of fluorescent dyes. This is followed by a more detailed consideration of both well established and more recently introduced neuroanatomical tracing methods. Technical aspects of the application, uptake mechanisms, intracellular transport of tracers, and the problems of subsequent signal detection, are also discussed. The methods that are presented and discussed in detail include: (1) anterograde and retrograde neuroanatomical labelling with fluorescent dyes in vivo, (2) labelling of post mortem tissue, (3) developmental studies, (4) transcellular tracing (phagocytosis-dependent staining of glial cells), (5) electrophysiological mapping combined with neuronal tract tracing, and (6) simultaneous detection of more than one axonal tracer. (7) Versatile protocols for three-colour labelling have been developed to study complex patterns of connections. It is envisaged that this review will be used to guide the readers in their selection of the most appropriate techniques to apply to their own particular area of interest. (C) 2000 Elsevier Science Ltd. All rights reserved.
\end{abstract}

Keywords: Neuroanatomy; Cell geometry; Anterograde and retrograde tracing; Fluorescent dyes; Multiple labelling; Electrophysiology

\section{Contents}

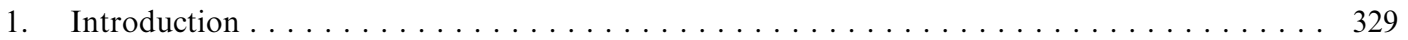

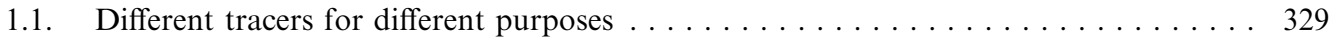

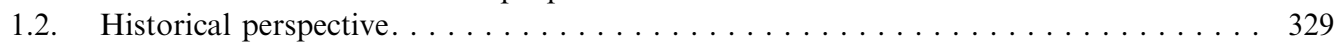

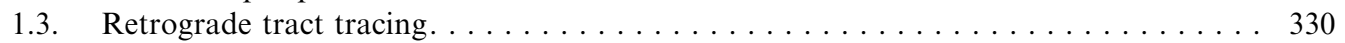

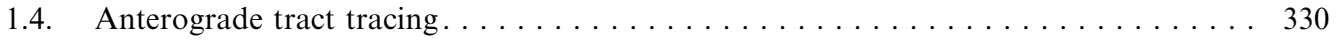

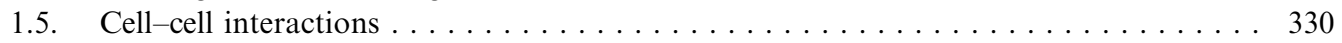

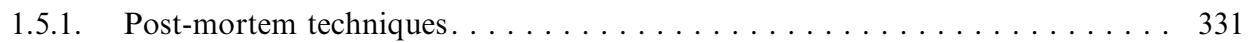

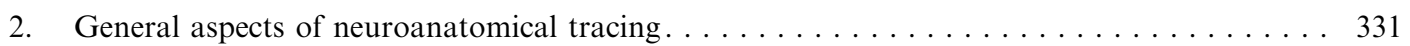

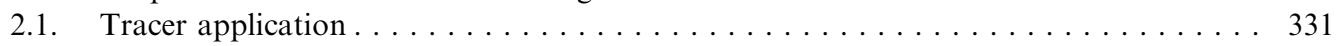

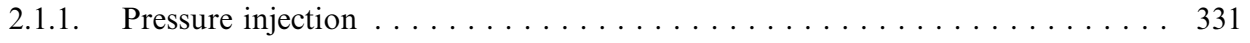

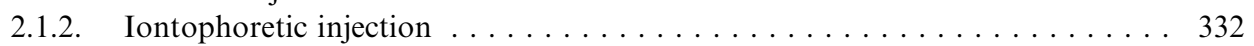

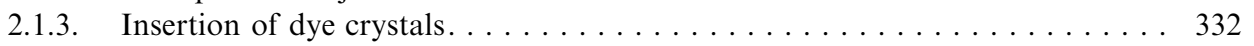

\footnotetext{
* Corresponding author. Tel.: + 49-251-8356-915; fax: +49-251-8356-916.

E-mail address: solon@uni-muenster.de (S. Thanos).
} 


\section{Nomenclature}

ABC avidin biotin peroxidase complex

BDA biotinylated dextran amine

BSI- $_{4} \quad$ Bandereia simplicifolia isolectin $\mathrm{B}_{4}$

CTB choleratoxin subunit b

DAB diaminobenzidine

DAPI diamidinophenylindol

DiA 4-4-dihexadecylaminostyryl- $N$-methyl-pyridinium

DiAsp 4-4-didecylaminostyryl- $N$-methyl-pyridinium iodide

DiI 1,1'-dioctadecyl-3,3,3',3'-tetramethyl-indocarbocyanine perchlorate

DiO 3,3'-dioctadecyloxacarbocyanine perchlorate

DMSO dimethylsulphoxide

DY Diamidino Yellow

EB Evans Blue

EC entorhinal cortex

EM electron microscopy

FB Fast Blue

FE Fluoro-Emerald

FG Fluoro-Gold
FITC Fluoresceine-isothiocyanate

FR Fluoro-Ruby

GFAP glial fibrillary acidic protein

HRP horseradish peroxidase

LM light microscopy

LY Lucifer Yellow

MR Mini Ruby

NY Nuclear Yellow

PAP peroxidase-anti-peroxidase

PBS phosphate-buffered saline

PFA paraformaldehyde

PHA-L Phaseolus vulgaris leucoagglutinin

PI propidium iodide

RGC retinal ganglion cell(s)

RITC Rhodamine- $B$-isothiocyanate

RT room temperature

TB True Blue

TBS Tris-buffered saline

TMB tetramethylbenzidine

TRITC tetramethylrhodamine isothiocyanate

TTC tetanus toxin fragment $\mathrm{C}$

WGA wheat-germ agglutinin

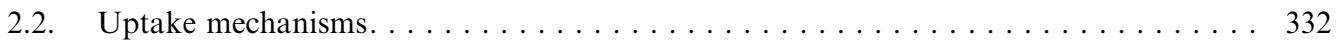

2.2.1. Active uptake . . . . . . . . . . . . . . . . . . . . . . . 332

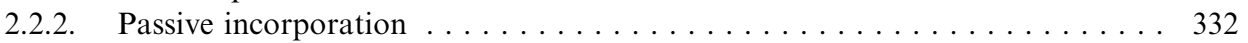

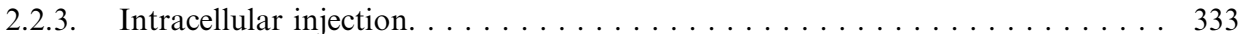

2.3. Transport and sequestration within the cells $\ldots \ldots \ldots \ldots \ldots \ldots \ldots \ldots \ldots$

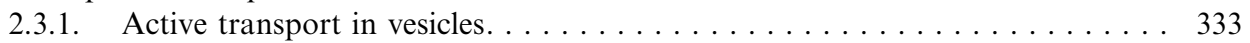

2.3.2. Lateral diffusion within the membrane . . . . . . . . . . . . . . . . 333

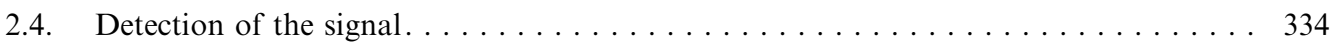

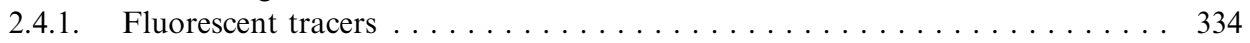

2.4.2. Non-fluorescent tracers . . . . . . . . . . . . . . . . . . . . . . . . . . . 334

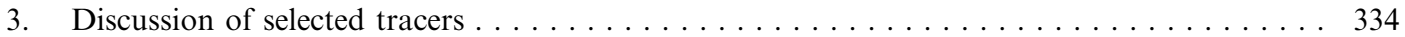

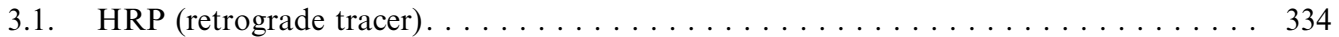

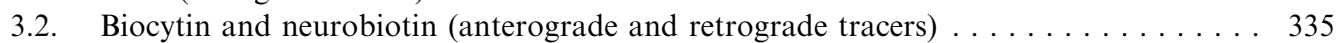

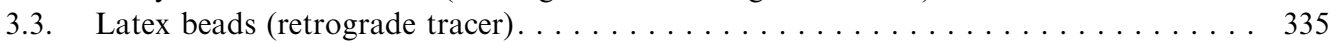

3.4. Plant lectins and bacterial toxins (anterograde and retrograde tracers) . . . . . . . 335

3.5. Fluorescent dyes (anterograde and retrograde tracers) $\ldots \ldots \ldots \ldots \ldots \ldots \ldots \ldots$

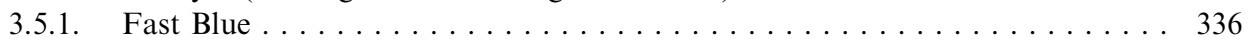

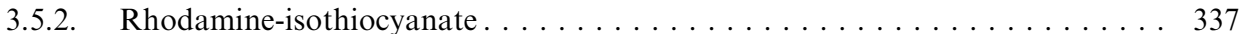

3.5.3. Carbocyanine dyes DiI, DiAsp, DiO, DiA . . . . . . . . . . . . . . 337

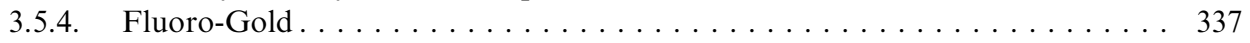

3.6. Dextran amines (predominantly anterograde tracers) $\ldots \ldots \ldots \ldots \ldots \ldots \ldots \ldots$

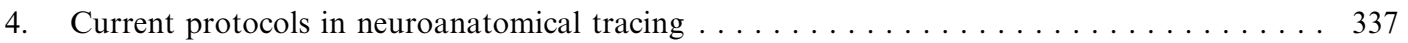

4.1. Neuroanatomical tracing with fluorescent dyes in vivo . . . . . . . . . . . 338

4.1.1. Anterograde and retrograde tracing with carbocyanines in the visual system . 338

4.1.2. Retrograde tracing of spinal cord motor neurones . . . . . . . . . . . . 338

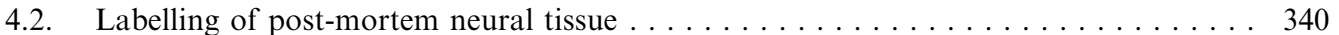

4.3. Developmental studies . . . . . . . . . . . . . . . . . . . . . . 340

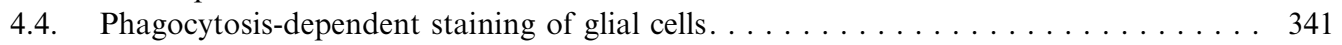

4.5. Electrophysiological mapping combined with neural tract tracing . . . . . . . . . . . 343 


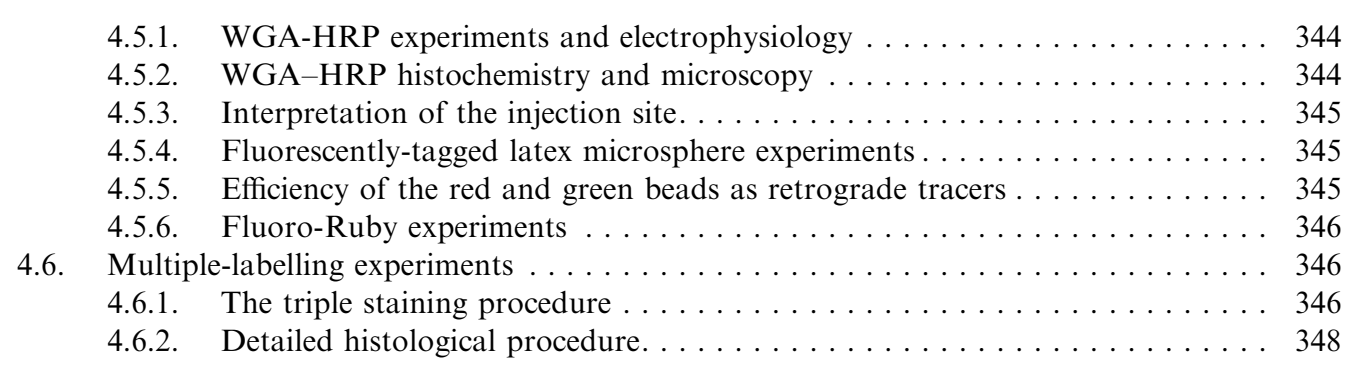

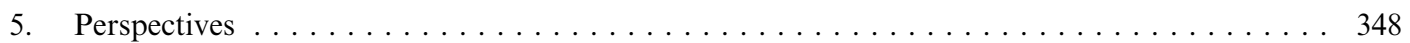

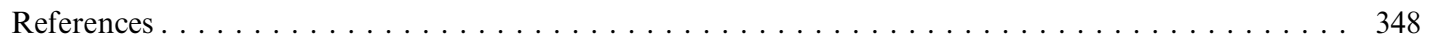

\section{Introduction}

\subsection{Different tracers for different purposes}

The fundamental purpose of neuronal tracing is to chart anatomical connections within the nervous system. Tracers are typically used to ascertain the cells of origin that innervate a certain brain structure, and the projection target of the axons of a given population of neurones. Modern neuroanatomical tracing strategies are not, however, confined to these problems because they can be combined with a variety of other techniques. In particular, in vivo labelling with fluorescent dyes allows not only the definition of neuronal populations according to their anatomical connections, but also makes them readily accessible to a wide range of additional approaches. These include:

- Immunohistochemical techniques (for instance, to characterise transmitter systems and the expression of given molecules).

- The morphological analysis of labelled cells, to allow the association between neuronal morphology and anatomical projection and/or functional status.

- Electron microscopy (EM) for ultrastructural characterisation.

- Prelabelling of neurones for their identification in cell culture, or for the additional use of a fluorescent cell sorter.

- The identification and collection of prelabelled cells for use in molecular biology.

- Electrophysiological approaches in brain slices or cell cultures, where the fluorescent labelling of projection neurones allows the identification of particular cells as targets for intracellular or patch-clamp recording.

- Developmental studies, to allow the investigation of mechanisms of axonal path finding in culture or in embryos.

- Multiple-labelling strategies using different fluorescent dyes in vivo or in fixed tissues to study divergent or convergent projections.
- Studies on human tissue than can only be assessed post mortem or after biopsy sampling.

- Analysis of nervous tissue from wild-living animals that cannot be assessed under laboratory conditions.

- Cell-lineage analysis during nerve cell migration.

- The genetic incorporation of fluorescent gene products into nerve cells (e.g., green or red fluorescent proteins).

These different roles demand a variety of properties to the tracers which will be considered in more detail in later sections.

\subsection{Historical perspective}

Over the last three decades, the step-by-step progress of tracing techniques has involved a range of different methodologies, all of which are based on a common principle, that of axonal transport. With regard to the direction of transport, it is possible to distinguish between the anterograde and retrograde transportation of tracer material. The discovery of the phenomenon of axonal flow by Weiss and Hiscoe (1948) has had far-reaching consequences, because it initiated decades of intensive study into the structural basis of this transport mechanism. In terms of neuroanatomical tools, retrograde axonal transport allows identification of the cells of origin of afferent nerve fibres to a particular target zone, while anterograde axonal transport enables the projection target(s) of individual or groups of cells to be charted within the central nervous system (CNS). For retrograde transport, the tracer material is applied to a fibre tract or a terminal field of innervation, becomes incorporated into the cell axons (usually by a process of endocytosis), and is then carried back to the parent cell body. For anterograde transport, the uptake mechanisms involve the cell soma and/or its dendrites, and the tracer material is transported along the axonal microtubular system to the cell's synaptic terminals. 


\subsection{Retrograde tract tracing}

The first attempts at retrograde tracing were fibre degeneration studies that were carried out in combination with impregnation methods (Fink and Heimer, 1967). Destruction of a target region of the brain leads to retrograde degeneration of the axons projecting to that region and, ultimately, to chromatolysis of the cells of origin of the pathway under study. Since degenerating fibres can be visualised with silver impregnation techniques, the course of the fibre tract can be charted from the lesion site along its length back to the cells of origin (Nauta and Gygax, 1954). A major breakthrough has undoubtedly been the discovery that the plant enzyme horseradish peroxidase (HRP) is taken up by axons and transferred back to cell somata by active retrograde transport. The enzyme reaction may then be used to stain the cell body and its major dendrites (Kristensson and Olsson, 1971; LaVail and LaVail, 1972; for a review see Mesulam, 1982). Subsequently, many new substances have been found that are useful for retrograde tracing. Of particular importance are fluorescent dyes of various colours, which can be combined in the same experiment for multiple retrograde labelling (Akintunde and Buxton, 1992).

The first attempts at retrograde labelling with fluorescent transport substances used Evans Blue dye (EB) combined with cattle albumin (see Kuypers and Huisman, 1984 for review). This prompted a "neuroanatomical revolution" that enabled the various advantageous properties of fluorescent dyes to tackle a huge range of questions. The number of dyes available and the fields of application have increased rapidly. The nuclear yellow marker diamidinophenylindol (DAPI), Fast Blue (FB), True Blue (TB), Nuclear Yellow (NY) and Lucifer Yellow (LY) are typical of those developed in the late 1970s. These have now been largely superseded by the use of Fluoro-Gold (FG), choleratoxin subunit b (CTB), fluorescently tagged beads and, most recently, Mini Ruby (MR) and fluorescently tagged dextran amines. Such developments have been motivated partly by whether or not the axon of a parent neurone can branch to more than one target. As a result, a range of different substances are available for double (and multiple) retrograde labelling studies, including modifications of various HRP techniques (see for example Kristensson, 1970; LaVail and LaVail, 1972; and Sections 5 and 6 of this paper).

\subsection{Anterograde tract tracing}

Anterograde tracing is used to trace the anatomical targets of a particular population of projection neurones. The tracer material is carried actively within the nerve fibres, but the transported substance moves from the cell body towards the axon terminals. During the 1970s neuroanatomical methods using anterograde tracing techniques rapidly replaced the histochemical detection of degenerating axons (lesion-induced Wallerian degeneration). Such techniques utilised the radioactively labelled amino acids (see Kuypers and Huisman, 1984 for a review) that contributed to the co-development of autoradiographic techniques. These are still used in a large number of applications. Perhaps the most famous example of these applications is the demonstration of ocular dominance columns (Hubel et al., 1977). The advantages of using tritiated amino acids are that they are readily incorporated into newly synthesised peptides, are transported across the synaptic cleft, and have a small molecular weight. The disadvantages include their hydrophilic properties, which limit their diffusion within the extracellular space, resulting in a restricted selectivity for certain cells or projections.

Subsequently, fluorescent dyes became available for anterograde tracing. At first, only a few were available, among them rhodamine-isothiocyanate (RITC) (Fig. 1) and 1,1'-dioctadecyl-3,3,3',3'-tetramethyl-indocarbocyanine perchlorate, (DiI; Thanos and Bonhoeffer, 1983, 1987). However, the list has since lengthened with the introduction of new classes of anterograde tracers including plant lectins, notably Phaseolus vulgaris leucoagglutinin (Gerfen and Sawchenko, 1984; Wouterlood et al., 1990a; Schofield, 1990; Ter Horst et al., 1984). More recently, dextran amines have become the most powerful tools for anterograde tracing (Glover et al., 1986) because they are relatively simple to use, and a great variety of detection methods have been developed for them. Dextrans can now be purchased in biotinylated form or conjugated to various fluorescent dyes, thus allowing immediate visibility or the production of permanent preparations after immunocytochemical processing.

\subsection{Cell-cell interactions}

The development of fluorescence techniques also led to the concept of cell-cell interactions and axonal guidance during development. Starting with the selective aggregation studies of dissociated cells from different origins, Hausmann and Moscona (1976) used the green fluoresceine-isothiocyanate (FITC) and the red dye RITC to label cells that aggregate because of their adhesive properties and the ability to recognise cells of the same origin. This led to the use of the same fluorescent dyes to identify selectively guided axons in culture (Bonhoeffer and Huf, 1980). Labelling of redfluorescent axons from the temporal retina were found to associate with co-cultured tectal membranes from the anterior tectum, and green-fluorescent axons were found to associate with posterior tectal membranes. In vivo studies soon followed, and for the first time 
RITC was used to study visual connections during development (Thanos and Bonhoeffer, 1983). A simple breakthrough was to apply solid crystals of slowly hydrolysing dyes such as RITC, to create a local reservoir at the selected site of injection. Retrograde tracers with hydrophilic properties, like FB and NY, were found to be transported anterogradely, but only over short distances (Kuypers and Huisman, 1984), and were therefore unsuitable for charting long-distance pathways. The introduction of the carbocyanine dyes DiI and 3,3'-dioctadecyloxacarbocyanine perchlorate (DiO) for vital retrograde (Honig and Hume, 1989) and anterograde staining (Thanos and Bonhoeffer, 1987) marked a significant new development by overcoming this problem. These tracers are convenient to use, since they are non-toxic, highly lipophilic, and can be applied as solid crystals. Growth-cone movement and axonal guidance have been studied extensively with carbocyanines, and their spectrum of application is ever increasing. Other fluorescent dyes with comparable intensity of staining, but higher solubility in aqueous solvents, are the low- and high-molecular-weight dextran amines (Fritzsch and Sonntag, 1991).

\subsubsection{Post-mortem techniques}

The development of the so-called "post-mortem" staining techniques soon followed, and the carbocyanines, most notably DiI, have been used for both anterograde (Godement et al., 1987) and retrograde labelling in fixed tissue (Vanselow et al., 1989). This lipophilic dye travels by lateral diffusion along the lipid membranes of the neurones. The advantages of using such a post-mortem technique are the facility to chart central connections within tissue obtained from wild-living animals and the ability to study embryonic or foetal tissue, which are otherwise inaccessible in utero. The application of these post-mortem techniques is, however, not limited to nerve cells, since glial cells (and possibly other cell types) can be stained through intramembraneous diffusion. One example of largescale screening with post-mortem staining is provided by its application in identifying pathfinding errors in the mutagenised zebrafish embryonic retinotectal projection (Trowe et al., 1996). Fluorescent probes are also used extensively in studies of cell-cell interactions in culture (e.g., Connors et al., 1984) or as voltage-sensitive dyes to detect changes in membrane potential (Grinvald et al., 1987; Zecevic and Antic, 1998). However, such applications are beyond the scope of this review, since they do not directly involve tracing along axons.

\section{General aspects of neuroanatomical tracing}

\subsection{Tracer application}

Three basic methods are used to deliver tracer material into the target tissue: pressure injection, iontophoretic injection and the mechanical insertion of dye crystals.

\subsubsection{Pressure injection}

The dissolved tracer material is usually delivered
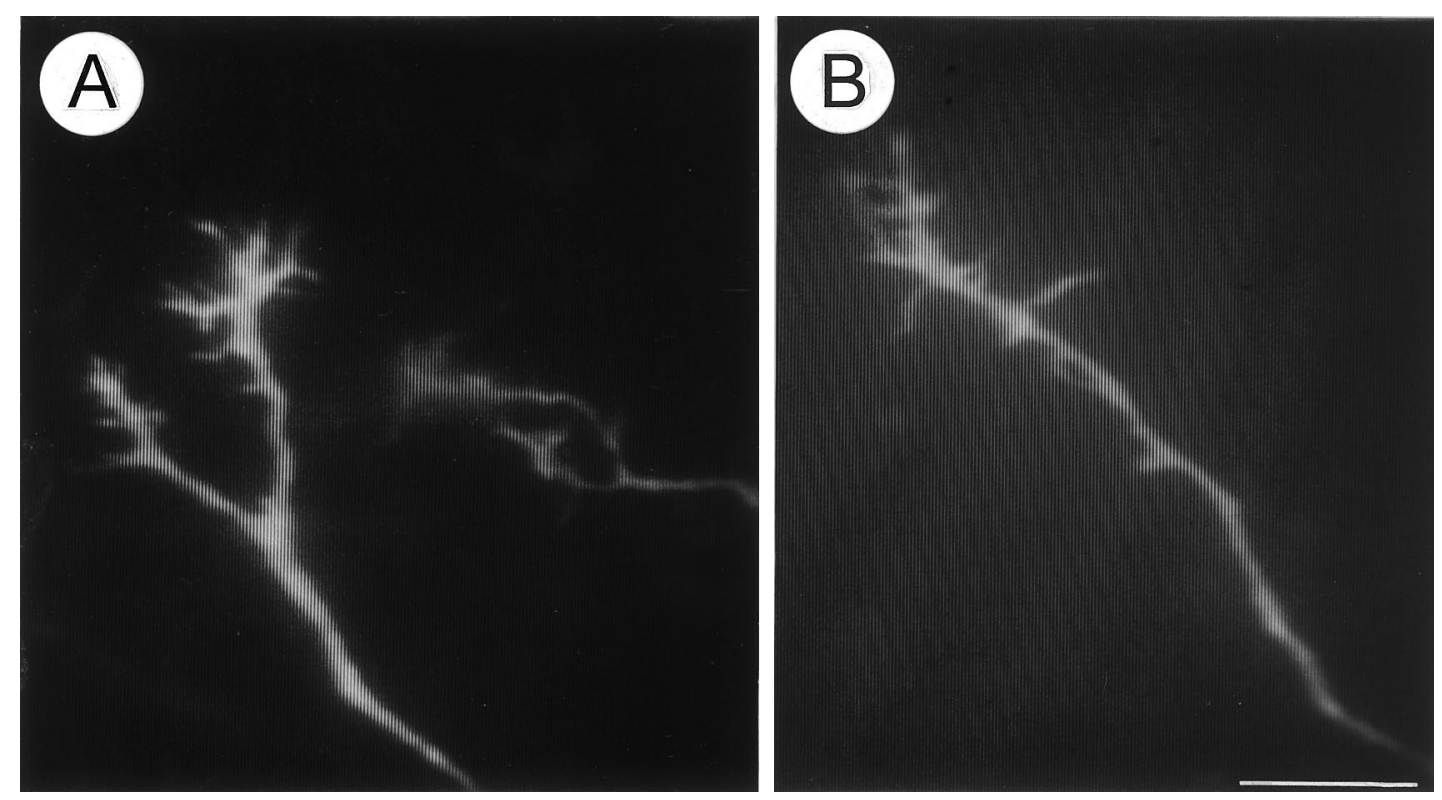

Fig. 1. A, B: Historically, one of the first indications of in vivo labelling of growth cones with anterogradely transported rhodamine- $B$-isothiocyanate. The dye was placed as a solid crystal into an embryonic chick retina and these photographs were taken from the living optic tectum 3 days later ( $\sim 8 \mathrm{~mm}$ distance) by using an image-intensifying TV camera. Note the details shown by the fluorescent dye. Scale bar: $10 \mu \mathrm{m}$. 
through a glass micropipette of the type used routinely in electrophysiological experiments and produced in a standard electrode puller. Injection of the tracer solution can be achieved either with gas pressure, or by means of a hydraulic system. The latter is often preferable because it enables the injection volume to be controlled accurately. The solvents required to prepare the tracer for injection vary depending upon the tracer. Some tracers are water-soluble, for instance FB or diamidino yellow (DY) and dextran amines, while others, like the carbocyanine dyes, require organic solvents. Suspensions of insoluble dyes must be sonicated and filtered prior to placing them into the micropipette. The concentration used also varies considerably between dyes, depending upon the sensitivity of the technique.

\subsubsection{Iontophoretic injection}

Tracer molecules usually carry an electrical charge and can therefore be ejected from the pipette by an applied electrical current. Current is applied through a micropipette containing the tracer solution (typically, at a concentration of about $1-10 \%$ ). With appropriate electrode tip diameters, this technique can be used for both intracellular and extracellular applications. For intracellular use the tracer is usually dissolved in $2 \mathrm{M}$ $\mathrm{KCl}$ or $2 \mathrm{M}$ potassium acetate $\left(\mathrm{KCO}_{2} \mathrm{CH}_{3}\right)$, and the injection current is typically 2-5 $\mathrm{nA}$ for $10-60 \mathrm{~min}$. The application time, (which is often a critical parameter) can be reduced greatly by using a low-molarity electrode solution. For example, complete intracellular fills of neurones with biocytin can be achieved after just 3 min with a $3 \mathrm{nA}$ current by using a solution of $0.5 \mathrm{M} \mathrm{KCO}_{2} \mathrm{CH}_{3}$. For extracellular iontophoretic application, electrode tips can be rather large, and the application current can be as high as $10 \mu \mathrm{A}$. An additional advantage of iontophoretic injections is that the application site can be precisely located prior to iontophoresis by conventional electrophysiological techniques, using the same micropipette that will deliver the dye.

\subsubsection{Insertion of dye crystals}

The insertion of crystalline dyes is feasible when the target tissue is normally readily accessible. As the labelling efficiency is very high and placement of the crystals can be carried out under microscopic control, this is the method of choice for focal applications of carbocyanine dyes such as DiI and 4-4-didecylaminostyryl$N$-methyl-pyridinium iodide (DiAsp). This type of method has also been used for the delivery of small quantities of other crystalline tracers, including wheatgerm agglutinin conjugated to HRP (WGA-HRP). The tissue penetration of the lipophilic carbocyanine dyes can be improved by adding a drop of incomplete Freund's adjuvant onto the crystal. The site of crystal- line dye insertion can be larger than $400 \mu \mathrm{m}$, making it possible to insert them into the deeper regions of the brain (see Section 4.4).

\subsection{Uptake mechanisms}

\subsubsection{Active uptake}

Tracers can enter axons or dendrites by active uptake via nerve terminals, or through severed neuritic profiles. In general both pathways work, but there are exceptions. For instance, dextran amines are taken up more efficiently by injured axons than by nerve terminals. Therefore, they are not suitable for neuronal tracing from muscle injections, and one has to keep in mind that fibres that traverse through an injection site (fibres en passage) will always be labelled as well. By contrast, propidium iodide (PI) and FB are more readily taken up via intact terminals than through disrupted axons. Such retrograde markers do not easily enter axons en passage and are therefore better suited for studies seeking to identify the origin of neurones terminating at the application site. Active uptake is one of the advantages of the lectins (Gerfen and Sawchenko, 1984). These plant-derived tracers bind to the sugar moieties of membrane-bound glycoproteins and glycolipids. Since receptor-mediated internalisation is very efficient, lower concentrations of tracer (between 1 and $5 \%$ ) suffice. For instance, WGA-HRP is internalised at a much higher rate than HRP alone.

\subsubsection{Passive incorporation}

Substances that diffuse passively into neurones enter because of the local concentration gradient. They must therefore be present at higher concentrations at the application site $(20-50 \%)$. Water-soluble tracers like HRP or FB reach the cytoplasm via the active transport of endosomes. Dyes that occur as weak bases diffuse through the membranes of intracellular vesicles. There, as a lower $\mathrm{pH}$ shifts amino groups to the protonised form, the lipophilic membrane becomes impermeable to the now-positively-charged molecules, and they accumulate within these vesicles. Uptake efficacy is determined by the permeability of membranes for the unprotonised form (Wessendorf, 1991). In this way, pH-trapping captures FG, TB, DAPI, NY and several other retrogradely transported molecules. Most fluorescent dyes are lipophilic substances. They are probably inserted into the outer leaflet of the cell membranes. Labelled membrane fragments become packed into vesicles and, as part of lipid turnover, are transported within the cell. This is the likely mechanism underlying the retrograde transport of DiI, DiO and DiAsp. The incorporation of lipids into membranes is efficient so that fluorescent dyes are generally given in low concentrations of $1-5 \%$. For many tra- 
cers, like fluorescent microspheres, the mechanism of uptake is still unknown.

\subsubsection{Intracellular injection}

If single cells are accessible for intracellular recordings, tracers can be delivered directly into the cytosol of a neurone. This method has been used extensively to visualise the cellular morphology with biotinylated dextran amine (BDA) or the fluorescent dye LY. Since intracellular iontophoretic injections are more applicable to brain slices in vitro, long-distance tracing is limited by the disruption of axons when the brain is cut up into slices. To the extent that projection targets are contained within a slice, anatomical connections can still be investigated using intracellular injections. For instance, this approach is being used to trace projections from the inferior colliculus to the optic tectum in the barn owl (Luksch et al., 1996). In some preparations intracellular injections are possible in vivo or in whole-brains in vitro, yielding the complete description of dendritic arborisations and the axonal projection of individual neurones (Luksch et al., 1996, 1998).

\subsection{Transport and sequestration within the cells}

\subsubsection{Active transport in vesicles}

There are two ways in which tracers are distributed within the neurones: active transport in vesicles and lateral diffusion within the plane of the membrane. Active vesicular transport seems to be the only efficient transfer mechanism for longer distances in vivo. By means of pH-trapping or the ingestion of labelled membrane fragments, the tracer accumulates in vesicles, and these are actively carried along the cytoskeleton of axons and dendrites. Fast axonal transport can reach velocities in the order of $2 \mathrm{~cm} /$ day. Whether transport occurs in the anterograde or retrograde direction depends upon the uptake mechanism and varies between tracer type. The anterograde transport of radioactively tagged proteins is somewhat slower, with velocities varying between 2 and $400 \mu \mathrm{m} /$ day. Retrogradely transported HRP, CTB and fluorescent markers that remain in vesicles produce a granular appearance in the cell soma, and consequently do not display the morphology of neuronal processes very well. Some hydrophilic substances, like FB, are more uniformly distributed within the cytoplasm. With membrane turnover, some lipophilic tracers, for example DiAsp (Mey and Thanos, 1993), or lectins that bind to particular membrane molecules seem to be reinserted into the membranes, thus leading to a much better labelling of cellular processes, which allows morphological analyses of the neurones labelled in this way. Tracers that have an affinity to nucleic acids (e.g., DY and NY) accumulate in the nucleus.

\subsubsection{Lateral diffusion within the membrane}

A second mechanism has been proposed for carbocyanine dyes that possess long lipophilic tails that insert into the cell membrane. The dyes spread through lateral diffusion within the plane of the membrane. This results in a much smoother labelling of the com-
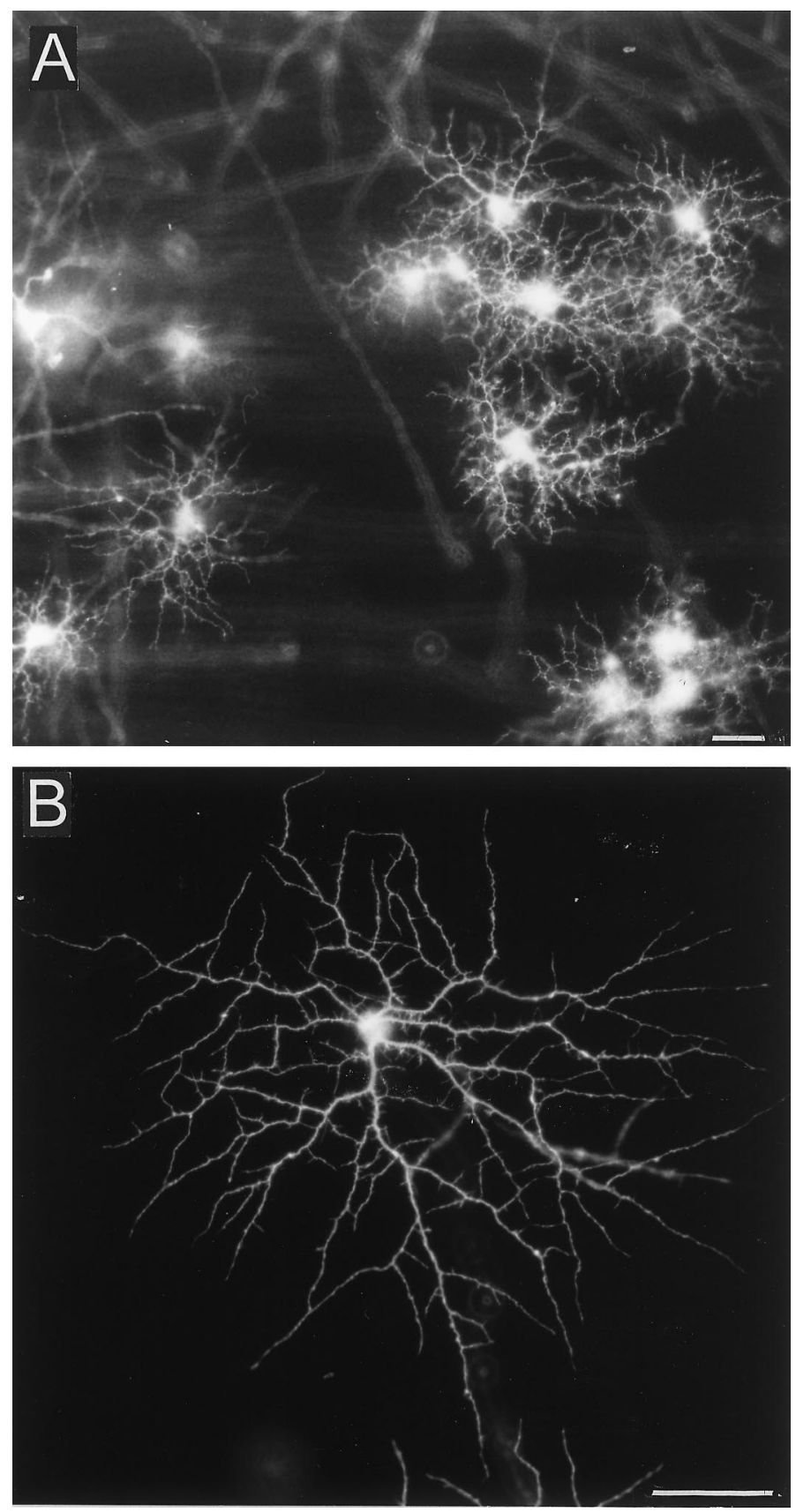

Fig. 2. Complete ganglion cell labelling with $1,1^{\prime}$-dioctadecyl$3,3,3^{\prime}, 3^{\prime}$-tetramethyl-indocarbocyanine perchlorate (DiI) in paraformaldehyde (PFA)-fixed retinal tissue. (A) Several ganglion cells are labelled in the vicinity of dye application within few days of incubation. (B) This larger magnification micrograph shows details of the cell morphology and dendritic ramification. Scale bars: $10 \mu \mathrm{m}$. 
plete extension of the cellular processes, and since it is based on passive diffusion this can be exploited for use in fixed tissue (Godement et al., 1987). Passive diffusion proceeds at a rate that is proportional to the temperature, at speeds of a few millimetres per day. Tracing after fixation with paraformaldehyde (PFA) can be performed faster when the tissue is kept in an incubator. The trade-off is reduced structural preservation. Depending upon the distance to be covered by the tracer and the importance one places on tissue quality, tracing in fixed tissues may be performed under refrigeration, at room temperature (RT), or at higher temperatures (e.g., $37^{\circ} \mathrm{C}$ ). Regardless of the temperature, the method is confined to tracing distances of a few centimetres (Fig. 2). After intracellular injections of LY or other markers, the entire cytoplasm is filled with tracer, which also allows complete staining of the cellular processes.

\subsection{Detection of the signal}

\subsubsection{Fluorescent tracers}

Fluorescent tracers are directly visible. This is a major advantage because labelled cells can be studied in the living tissue in vivo or in vitro, and it is feasible to perform additional experiments on the same tissue, for instance electrophysiology or the observation of growth processes in culture. However, a relative disadvantage is that fluorescent dyes are frequently not preserved in cryostat or paraffin sections. This can be overcome by using a vibratome or tissue chopper. There is now a large number of fluorescent tracers available with different absorption spectra, thus permitting simultaneous labelling with multiple markers. These advantages of fluorescent dyes are offset by a few drawbacks. A strong fluorescence signal may obscure the details of smaller processes. Photobleaching, or fading of the signal after light exposure during examination, poses a problem to the application of any fluorescent marker. This is severe for some substances (e.g., fluoresceine) and less of a problem for others (DiAsp, DiI, FG). Since photobleaching is believed to be caused by oxygen and oxygen-induced free radicals, antioxidants reduce fading. One successful formula, which can be used as a mounting medium, is $0.1-0.25 \mathrm{M} n$-propyl gallate in glycerol (Giloh and Sedat, 1982). For fluorescently labelled latex beads, commercially available mounting media like Fluoromount must be used rather than glycerol. To achieve a permanent staining the fluorescence can be converted into a dark, electron-dense material, which precipitates after incubation with diaminobenzidine (DAB). This reaction requires great caution since the $\mathrm{DAB}$ solution, which has to be illuminated for some time with the aid of a fluorescence microscope, is toxic, carcinogenic, and sticks to contaminated surfaces. Some fluorescent tracers diffuse out of the cells and are therefore not suitable for long-term studies, but this is not a problem with most carbocyanine dyes. In addition, frequent illumination of short-wavelength fluorescence (e.g., FB) leads to phototoxicity of otherwise nontoxic substances. And finally, since some fixatives have autofluorescent properties, tissue fixation cannot be optimised. Glutaraldehyde, for instance, should not be used with fluorescent tracers because of its autofluorescence.

\subsubsection{Non-fluorescent tracers}

Most other tracers can be detected with specific antibodies that may be visualised with the usual immunohistochemical methods. There is a whole battery of techniques that can be employed in combination with neuroanatomical tracers, including signal amplification with secondary antibodies, indirect methods like the peroxidase-anti-peroxidase (PAP) method, avidin-biotin-peroxidase $(\mathrm{ABC}) \mathrm{kits}$, and detection with peroxidase or alkaline phosphatase reaction. Progress in routine immunohistochemical methods has therefore also improved the sensitivity of neuroanatomical tracing. Dextran amines, for instance, are conjugated with biotin so that they can be detected via the highaffinity binding of avidin or streptavidin that has been labelled with a substrate of some kind (e.g., HRP or a fluorescent tag). The tracers biocytin and neurobiotin are direct receptors of avidin. Antibodies are also available for some of the fluorescent dyes like FG and LY. The combination of tracing studies with ultrastructural investigations involves the production of an electron-dense DAB precipitate after photoconversion of fluorescence or gold-labelled secondary antibodies.

\section{Discussion of selected tracers}

\subsection{HRP (retrograde tracer)}

The plant enzyme HRP was the first retrograde neuroanatomical tracer to be used. It may be pressure injected using $20-50 \%$ solutions in $2 \%$ dimethylsulphoxide (DMSO), iontophoresed or applied as lyophilysed enzyme. Usually, the tracer is driven out of micropipettes that are used for simultaneous electrophysiological recordings, by gas pressure or negative currents. In some cases the use of large amount of concentrated tracer compromises precision. Uptake by nerve terminals occurs via the cytoplasm into vesicles where the enzyme resides. Detection of the tracer molecules takes advantage of its enzymatic properties. Since the brown precipitate that is produced after the reaction of HRP with hydrogen peroxide and either $\mathrm{DAB}$ or tetramethylbenzidine (TMB) is stable and electron-dense, HRP-labelled neurones are amenable 
to ultrastructural analysis. As a major drawback, however, the staining produced using HRP is incomplete, usually being limited to the cell soma and primary dendrites. This method of detection does not permit vital staining (Kristensson and Olsson, 1971; Mason et al., 1988; Schütte and Hoskins, 1993).

\subsection{Biocytin and neurobiotin (anterograde and retrograde tracers)}

Biocytin and biotinamide (neurobiotin) were introduced as intracellular markers for electrophoresis through glass micropipettes. For anatomical tracing, both compounds can be injected by extracellular iontophoresis with high currents and large bore pipettes, and are applicable for both anterograde and retrograde tracing. Intracellular transport is very effective and leads to labelling of the finest axonal arborisations. Compared to other tracers, biocytin and neurobiotin are relatively small (molecular weight of 372 $\mathrm{kDa}$ and $286 \mathrm{kDa}$, respectively) and, as their major advantage, have a high affinity for avidin, which provides the basis for their detection. After tracing in vivo, brains are usually perfused, sectioned, and processed with an $\mathrm{ABC}$ kit, and then subjected to the enzymatic reaction with DAB. As with HRP, this procedure enables the performance of ultrastructural studies and can be combined easily with immunohistochemistry, but it precludes visualisation of the tracer in culture or living tissue. However, a variety of fluorescent dyes coupled to avidin or streptavidin is now commercially available, thus allowing a fluorescent visualisation of the biotin derivatives for double-labelling experiments (Horikawa and Armstrong, 1988; Kita and Armstrong, 1991; Lapper and Bolam, 1991; Wirsig-Wiechmann, 1994).

\subsection{Latex beads (retrograde tracer)}

Fluorescent latex microspheres contain red or green fluorescent material, with the signal produced by the rhodamine-filled red beads being superior to that produced by the fluoresceine-filled green beads. The beads, which are prepared in an aqueous solution, are pressure injected into the selected site. To trace local connections, very small volumes $(30-50 \mathrm{nl})$ of bead suspension are injected through glass pipettes that have a tip diameter of $30-50 \mu \mathrm{m}$. For long-range retrograde tracing, larger volumes (up to $0.5 \mu \mathrm{l}$ ) and larger pipette tips or $1-\mu 1$ Hamilton syringes may be used. While the uptake mechanism for this tracer is not completely understood, transport occurs in vesicles exclusively in the retrograde direction. For most axonal connections survival times of $24-48 \mathrm{~h}$ suffice, but labelling does not deteriorate in either quality or extent after survival times of several months, and no cell leakage occurs. Since retrograde transport is fast and reliable, fluorescent beads have become the axonal tracer of choice when anatomically defined neurones are to be identified for additional experiments in vivo. Since microspheres are visible only within the cell soma, most fluorescent dyes provide a superior signal. With respect to signal detection and processing of the tissue, most precautions concerning fluorescent signals apply. In addition, these beads are very sensitive to glycerol and will fade rapidly if mounted in glycerolcontaining solutions. It is possible to make frozen sections of bead-labelled tissue. Since exposure to xylenes or alcohols for more than 5 min destroys the beads, the clearing of air-dried slides must be kept to a minimum. Attempts to retain bead labelling after plastic embedding have not been successful (Katz and Iarovici, 1990; Katz et al., 1984; Senatorov et al., 1997)

\subsection{Plant lectins and bacterial toxins (anterograde and retrograde tracers)}

Plant lectins and related bacterial toxins are characterised by their high affinity to specific sugars. After binding to glycoconjugates of the neuronal membrane, they are internalised and transported within the neurites of many neurones. WGA was the first lectin introduced for neuronal tracing. When conjugated to HRP, it greatly enhanced the uptake and transport of this molecule. Since then, many more lectins and bacterial toxins with different binding specificities, and varying affinities to different neuronal populations, have been discovered. The following list is far from comprehensive:

\begin{tabular}{|c|c|c|}
\hline Lectin/toxin & $\begin{array}{l}\text { MW } \\
(\mathrm{kDa})\end{array}$ & Binding specificity \\
\hline WGA & 36 & $\begin{array}{l}N \text {-Acetyl-glucosamine } \\
\text { and sialic acid }\end{array}$ \\
\hline Peanut agglutinin & 110 & $\beta$-D-Galactose \\
\hline Concanavalin A & 104 & $\alpha$-D-Mannose \\
\hline $\begin{array}{l}\text { Phaseolus vulgaris- } \\
\text { leucoagglutinn } \\
\text { (PHA-L) }\end{array}$ & & $\begin{array}{l}\alpha-D-M a n n o s e \\
\beta \text {-D-galactose }\end{array}$ \\
\hline Lens culinaris & $42-60$ & $\alpha$-D-Mannose, $\beta$-D-glucose \\
\hline Soy bean agglutinin & 120 & $N$-Acetyl-galactosamine \\
\hline $\begin{array}{l}\text { Ulex europaeus } \\
\text { agglutinin }\end{array}$ & $43-170$ & $\alpha$-L-Fucose \\
\hline $\begin{array}{l}\text { Rhicinus communis } \\
\text { agglutinin I }\end{array}$ & 120 & Lactose and $\beta$-D-galactose \\
\hline $\begin{array}{l}\text { Rhicinus communis } \\
\text { agglutinin II }\end{array}$ & 60 & $\begin{array}{l}\text { Lactose and } \beta \text {-D- } \\
\text { galactose- }(1,3)-N \text {-acetyl- } \\
\text { galactosamine }\end{array}$ \\
\hline CTB & 12 & Monosialoganglioside \\
\hline Tetanus toxin, & 47 & Trisialoganglioside \\
\hline
\end{tabular}

fragment C (TTC) 


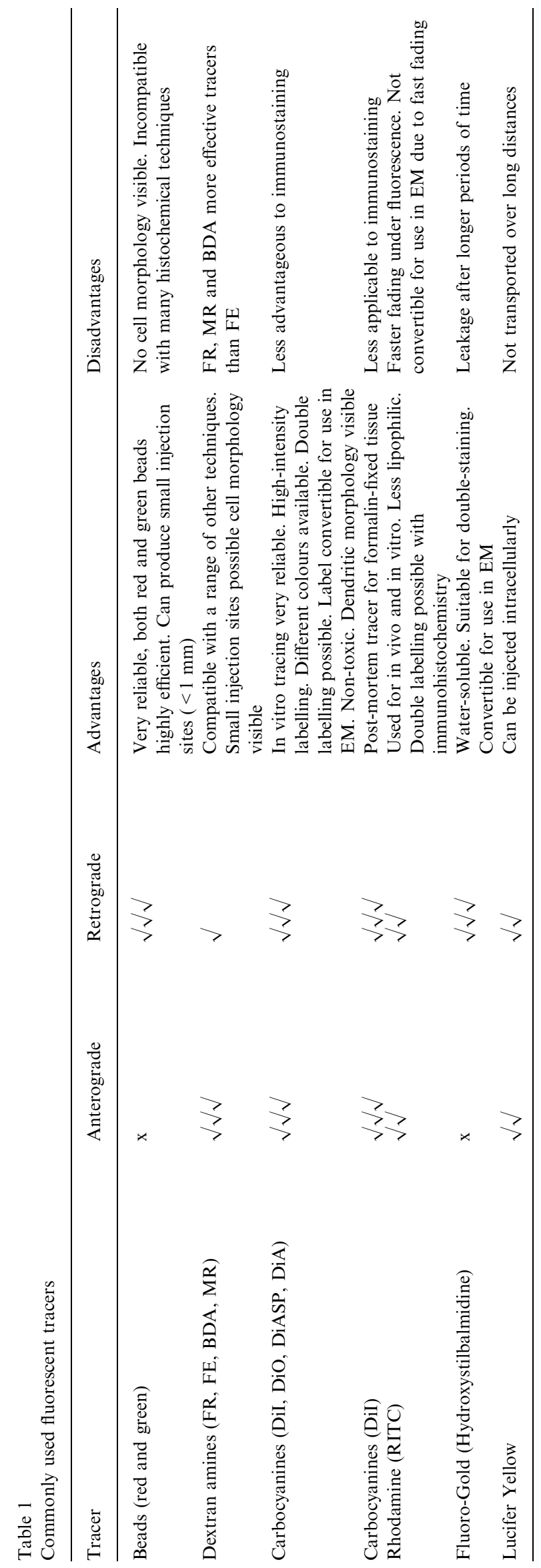

It is sometimes possible to reduce toxicity by using only parts of the molecules. Lectins and toxins are used for both retrograde and anterograde applications. In fact, PHA-L is one of the earliest and most frequently used anterograde tracer. Some of these molecules, TTC for instance, are transported across synapses into other neurones, and are also incorporated by fibres en passage. Lectins and toxins may be applied using all of the in vivo injection techniques described previously. For detection, some lectins are biotinylated so that they can be used in avidin-binding protocols, or they may be conjugated to HRP. They can then be visualised either enzymatically or immunohistochemically. They are also available coupled to fluorescent dyes. Many lectins and toxins can be detected with highly specific antibodies (Cabot et al., 1991; Gerfen and Sawchenko, 1984; Sawchenko and Gerfen, 1985; Schofield, 1990; Ter Horst et al., 1984; Wouterlood et al., 1990b).

\subsection{Fluorescent dyes (anterograde and retrograde tracers)}

Fluorescent dyes are probably the most frequently used neuroanatomical tracers. The first ones to be employed directly for anatomical tracing were PI, NY (Björklund and Skagerberg, 1979), RITC and FB (Bonhoeffer and Huf, 1980; Bentivoglio et al., 1980). In 1986, Honig and Hume introduced the carbocyanine dyes DiI and DiO. It was also in 1986 that FG came into use. Examples of frequently used fluorescent tracers are given below, and some of the key features of the most widely used fluorescent tracers are summarised in Table 1.

\subsubsection{Fast Blue}

FB is applied by pressure injection of a $3 \%$ aqueous solution. It is taken up by axon terminals rather than by injured axons. Retrograde tracing leads to uniform labelling of the cell soma without much staining of dendrites. The blue fluorescence requires excitation in the ultraviolet range $(360 \mathrm{~nm})$ and is subject to high phototoxicity at longer illumination times. This problem is common to other blue dyes like TB and Granular Blue (GB), which are less efficient than FB. In culture conditions, this dye has also been found to interfere with cell adhesion, migration and proliferation. Longer survival times increase fluorescence, but then dye leakage occurs, resulting in staining of surrounding glial cells and a decrease in the number of labelled neurones. In this respect other dyes with similar applications [FG, Mini Ruby (MR), Fluoro-Ruby (FR), Fluoro-Emerald (FE)] are even worse than FB (Akintunde and Buxton, 1992; Bentivoglio and Su, 
1990; Bentivoglio et al., 1980; Novikova et al., 1997; Vince et al., 1997).

\subsubsection{Rhodamine-isothiocyanate}

Rhodamine serves as both a retrograde and anterograde tracer. It emits red fluorescence (>590 nm) after excitation with green light at a maximum wavelength of $545 \mathrm{~nm}$. It can be pressure injected at a concentration of $1-3 \%$ in an aqueous solution containing $1 \%$ DMSO or $0.2 \%$ ethanol. Alternatively, crystals may be inserted into the target tissue. RITC is much more effective than FB, as evidenced by the number of neurones that can be labelled, and is also retained within labelled neurones without leakage over very long periods. Phototoxicity poses no problem in cell culture. In retrograde tracing experiments, RITC fluorescence was found to be as bright and more evenly distributed than that of the carbocyanine dyes (Thanos and Bonhoeffer, 1983, 1987). The green-fluorescence dye FITC can be used in conjunction with RITC to double label cells from different putative projection sites, for example. However, the fluorescence emitted by FITC fades faster than that emitted by RITC, and it is less suitable for staining in vivo.

\subsubsection{Carbocyanine dyes DiI, DiAsp, DiO, DiA}

These tracers are highly lipophilic, providing the advantage that they diffuse through membranes even in fixed tissue. However, this property also limits their application in vivo. For pressure injection, dyes are dissolved in organic solvents, for instance $2.5 \%$ in a $1: 1$ solution of ethanol/DMSO, or $3 \%$ in dimethylformamide. Focal applications are often made by inserting crystals of these dyes, a technique that is also suitable for tract tracing in fixed tissue. When incorporated into vesicles with labelled membrane fragments, carbocyanine dyes are transported actively in both directions (anterograde and retrograde). No transfer occurs between cells, and the dye remains viable for long periods of time. The dyes are available in various colours (DiI, fast DiI: red; DiAsp: yellow; 4-4-dihexadecylaminostyryl- $N$-methyl-pyridinium DiA, DiO: green) and give a high fluorescence yield with little fading. The efficacy of several of these molecules has been compared in the mammalian and avian visual systems (Godement et al., 1987; Hong and Thanos, 1996; Honig and Hume, 1989; Mey and Thanos, 1993; Veenman et al., 1992; see Section 4.1.1). The labelling is stable after survival times of up to 1 year.

\subsubsection{Fluoro-Gold}

When dissolved to a concentration of $1-10 \%$ in buffer, this yellow-golden dye can be applied via iontophoretic injections or with pressure. After incorporation by injured axonal profiles and terminals the dye accumulates in vesicles, is transported in a ret- rograde direction, and often gives a granular appearance to the labelled cell somata. Hydroxystilbamidine, which has been identified as the active component of FG, has different optical characteristics depending upon the $\mathrm{pH}$. Under physiological conditions, the best excitation occurs at $325 \mathrm{~nm}$, when the emitted light has a maximum wavelength of $440 \mathrm{~nm}$. Antibodies raised against FG are also available, which means that the dye can be converted into a permanent substrate and that it is possible to plastic embed sections containing FG-labelled tissue without losing the tracer. Despite its excellent tracing properties, FG is not suitable for long-term labelling where survival times of several months are required (Novikova et al., 1997; Schmued and Fallon, 1986; Schmued et al., 1990; Wessendorf, 1991).

\subsection{Dextran amines (predominantly anterograde tracers)}

The water-soluble dextran conjugates can be applied by any of the injection techniques or as crystals, and air-dried onto needles. Compared to most other fluorescent dyes like FG, carbocyanines and RITC, dextrans have high molecular weights $(10 \mathrm{kDa})$. They are incorporated into injured neurones at the injection site and are transported rapidly in a predominantly anterograde direction. Uptake can be enhanced by co-injection of permeabilising agents like 5\% Triton X-100. The dextrans are probably among the best neuroanatomical tracers available since they label cellular processes in much detailed fashion. In addition, the tracers can be detected with a variety of methods, for example they can be conjugated to fluorescent dyes or biotinylated, and are suitable for EM without prior alteration. However, when the persistence of the labelling attained using dextran amines is compared with that of several other tracers, it appears that dextran conjugates, like FG, are more suitable for short-term experiments (Brandt and Apkarian, 1992; Glover et al., 1986; Nance and Burns, 1990; Novikova et al., 1997; Rajakumar et al., 1993; Veenman et al., 1992; Wouterlood and Jorritsma-Byham, 1994).

\section{Current protocols in neuroanatomical tracing}

A detailed description of a variety of neuroanatomical tracing methods is presented below, to provide an insight into the ways in which some of the neuroanatomical techniques mentioned in this paper are currently being used. 


\subsection{Neuroanatomical tracing with fluorescent dyes in vivo}

\subsubsection{Anterograde and retrograde tracing with carbocyanines in the visual system}

The vertebrate visual system is one of the most suitable models for examining the ontogeny of neuronal projections, degenerative or regenerative processes, and for the determination of cell fate in the CNS. Indeed, the suitability of several carbocyanine dyes for anterograde and retrograde tracing has been compared using the mammalian (rat) and avian (chick) visual systems as models (Mey and Thanos, 1993; Thanos and Mey, 1992, 1995; Thanos et al., 1994).

The insertion of dye crystals into the retinal surface has allowed the anterograde tracing of axonal projections from retinal ganglion cells up to the retrobulbar area of the brain. In order to label the complete retinofugal projection, it was found to be more useful to inject a tracer solution into the vitreous body. For anterograde tracing in the embryonic chick, the best results were obtained with DiI. Anterograde tracing with this carbocyanine revealed the finest axonal arborisations, while with a similar rate of transport, retrograde tracing with DiI and DiA produced only granular labelling without displaying the dendrites. Using DiAsp, the retrograde tracing of retinal ganglion cells (RGCs) and their dendrites from the superior colliculus and the optic nerve of the rat produced the finest labelling of dendrites, superior to that produced by FG and the other carbocyanine dyes.

One of the staining techniques used to retrogradely trace RGCs from the optic nerve in rats, was performed by Thanos et al. (1994), for review, as follows. The optic nerve of the deeply anaesthetised rat was exposed intraorbitally and, after longitudinal incision of its meningeal sheath, the nerve was completely transected about $1 \mathrm{~mm}$ from the eye bulb without affecting the retinal blood supply. Crystals of DiAsp were applied to the cross-sectional surface of the nerve and the labelled nerve stump was surrounded with resorbable sponge (Gelfoam, Upjohn, Denmark) to prevent displacement of the dye crystals. After suturing of the skin wound the animals were returned to their cages for at least 3 days to allow for retrograde transport of the dye and complete labelling of RGCs to occur. After this recovery period the animals were killed and their eyes were removed. The retinae were dissected and spread onto black nitrocellulose filters (Sartorius, Göttingen) with the ganglion cell layer upwards. The mounted retinae were fixed in $4 \%$ PFA (in $0.1 \mathrm{M}$ phosphate-buffered saline, PBS) and viewed as wholemounts through the fluoresceine filters of a fluorescence microscope.

\subsubsection{Retrograde tracing of spinal cord motor neurones}

For the retrograde tracing of spinal cord motor neurones, one of four different application techniques may be utilised:

- Injection of a dye solution into the muscle (Kristensson, 1970; Richmond et al., 1994)

- Pressure injection of a dye solution into the proximal nerve stump followed by ligature of the nerve stump to prevent dye leakage (Schmid et al., 1983).

- Incubation of the transected nerve stump in small containers filled with a dye solution (Harsch and Madison, 1991).

- Application of dye crystals onto the transected or incised nerve stump (Fritzsch and Sonntag, 1991; Novikova et al., 1997).

This latter technique will be described here because it results in a high labelling efficiency, is relatively easy to perform, and can be adapted to many experimental systems. This method is applicable to those dyes that are lipophilic and remain in the nerve over a longer period of time after surgery. It provides the advantages of reaching a maximal concentration at the site of deposition and of being applicable to defined positions in the nerve. As shown by Köbbert and Thanos (2000), DiI, and to a lesser extent other (carbocyanine) dyes, seems ideal for experimental approaches that deal with nerve topography (Fig. 3).

The sciatic nerve can be exposed easily. The skin is incised at the level of the hip and femur, and the muscles are carefully prised apart at the caudal border of the M. gluteus maximus, revealing the sciatic nerve, which can be dissected without damaging any muscle fibres. The connective tissue surrounding the nerve is carefully removed and the nerve is transected at the mid-thigh level (about $1 \mathrm{~cm}$ distal from the hip joint). Fluorescent dyes with different excitation wavelengths and physico-chemical properties, and which are known to be non-toxic, are selected as local tracers from the site of the sciatic nerve lesion. The carbocyanine dyes, DiI, DiO (Honig and Hume, 1989; Novikova et al., 1997; Richmond et al., 1994), and DiAsp (Thanos et al., 1994 for review) and FG (Schmued and Fallon, 1986) may be used as tracers. Small crystals of the dye are placed onto the proximal nerve stump and held in place with resorbable gelfoam.

After a survival period of 10-14 days (depending upon the transport rates of the different tracers used), the rats are re-anaesthetised and perfused transcardially with phosphate-buffered saline (0.1 M PBS, pH 7.4) followed by fixative (4\% PFA in $0.1 \mathrm{M}$ PBS, $\mathrm{pH}$ 7.4). The lumbosacral spinal cord is exposed after removing the skeletal muscles. The vertebrae and the dura mater from T11 to L3 are carefully removed. The now easily accessible lumbar enlargement is removed and postfixed overnight at RT. 
To enable the correct orientation of transverse spinal cord sections, a longitudinal incision is made along the entire length of the left side of the spinal cord. The lumbar enlargement is embedded in mounting medium and frozen in liquid nitrogen. Since motor neuronal somata have a large diameter (15-70 $\mu \mathrm{m}$; Neuhuber and Niederle, 1979), 50- $\mu \mathrm{m}$-thick serial transverse sections can be made. These sections are collected in PBS to wash out the mounting medium and remnants of the fixative. They are mounted in serial order on gelatinised slides, air dried and coverslipped using Moviol (Merck), which is a non-fluorescent mounting medium. The sections can be examined directly with the aid of a microscope equipped with an epifluorescence system. The following filter systems are used to visualise the four different fluorescent tracers: rhodamine filters [bandpass (BP 546) fluorescence threshold (FT 580) longpass (LP590)] for DiI, fluoresceine filters (BP 450490/FT 510/LP 520) for DiAsp and DiO, and ultra- violet filters (BP 365/FT 395/LP 397) for FG. The distribution of retrogradely labelled motor neurones can be documented photographically (using any commercially available high-speed film), as shown in Fig. 3.

The retrograde markers have to cover a distance of approximately $6-7 \mathrm{~cm}$ in adult rats (female rats, weighing approximately $200 \mathrm{~g}$ ) in order to label the sciatic nerve motor neurones between the L4 and S2 segments. Of the dyes tested, DiI has been shown to label perikarya throughout the lumbosacral region 8-9 days after dye-crystal deposition into the sciatic nerve (Köbbert and Thanos, 2000). The fluorescence appears bright red and granular, and remains confined to the extra-nuclear cytoplasm (Fig. 3A). Fading after fluorescence illumination is negligible, and the dye does not diffuse or leak out of the cells. The green dye $\mathrm{DiO}$ is transported slightly slower than DiI, and stains the cytoplasm only weakly (Fig. 3B). In addition, the fluorescence attributable to $\mathrm{DiO}$ fades rapidly upon ex-
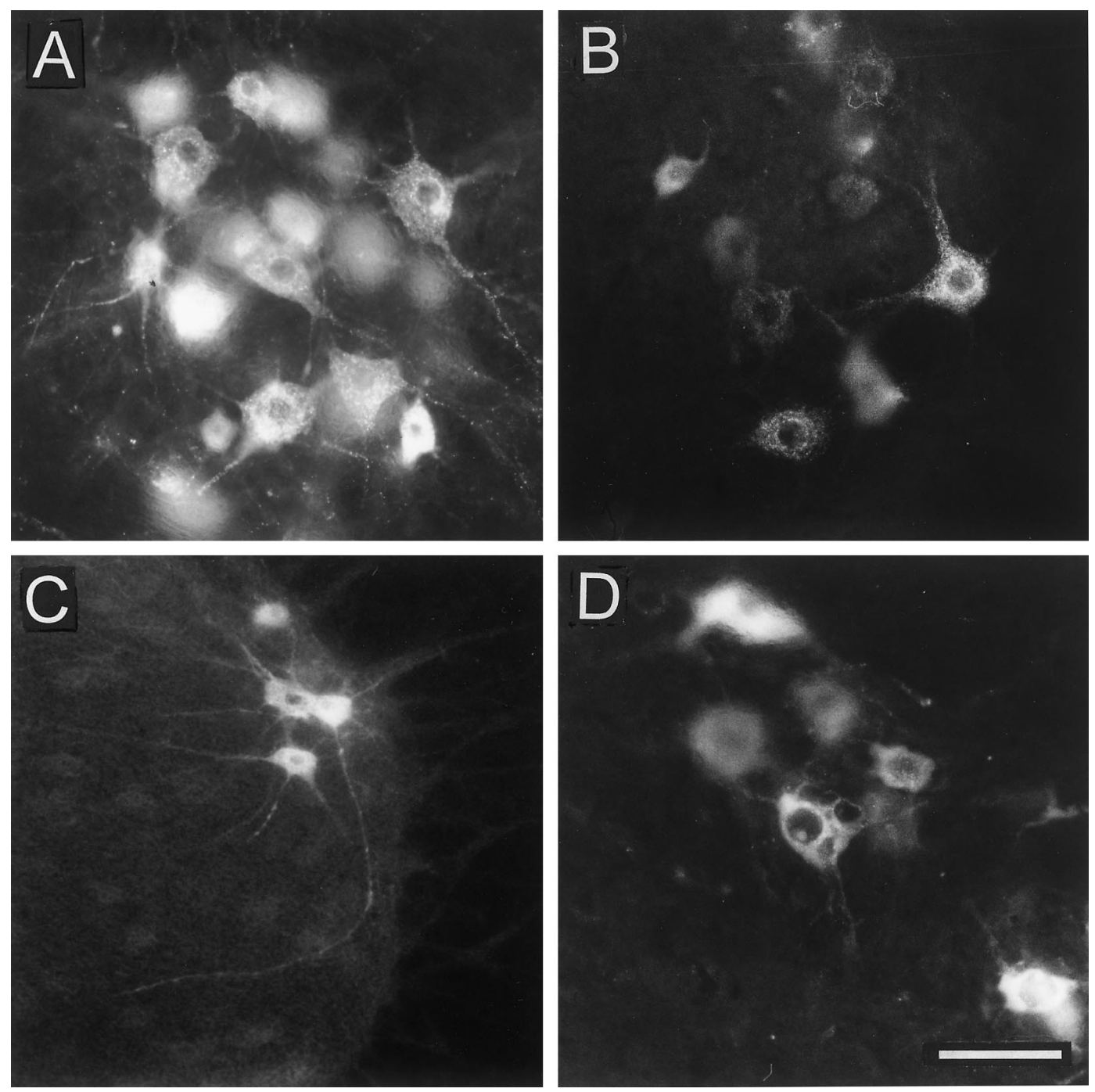

Fig. 3. Typical appearance of fluorescent spinal cord motor neurones after retrograde tracing with (A) DiI, (B) DiO, (C) DiAsp, and (D) FluoroGold (FG) from the sciatic nerve. Note the fluorescent nucleolus in the FG-labelled neurones. Scale bar: $100 \mu \mathrm{m}$. 
posure to the blue excitation light. These features make DiO less suitable for these experiments than DiI (Köbbert and Thanos, 2000). The third carbocyanine dye, DiAsp, is transported slightly slower and labels the cytoplasm, the proximal dendrites and axons with a bright yellow fluorescence 14 days after deposition in the sciatic nerve (Fig. 3C). However, this dye leaks out of the motor neurones after they are sectioned and forms a perineuronal "halo", blurring the motor neurone outlines. FG renders a whitish-yellow fluorescence to the cytoplasm, does not leak, and also labels the nucleoli in most intensely stained neurones (Fig. 3D). On average, FG labelling is less intense than DiI and DiAsp but, like DiI, it is resistant to fading.

\subsection{Labelling of post-mortem neural tissue}

Carbocyanine dyes (DiI, DiO, DiA, DiAsp) exhibit a strong and sometimes voltage-dependent light-excitable fluorescence. The chemical structure of these dyes and their highly lipophilic properties permit them to become inserted in the lipid fraction of the plasma membrane of intact cells to diffuse freely within the membrane (Waggoner, 1976). In addition to their application as tracers in living tissue, the carbocyanine DiI was the first dye to be used in fixed neuronal tissue (Godement et al., 1987; Vanselow et al., 1989). This staining can be performed in a convenient way and after various methods of fixation, with slight advantages afforded to tissue fixed with $2-4 \%$ phosphatebuffered formaldehyde. Pre-fixed neural tissue en block, sliced, or whole-mounted, can be used either immediately after the onset of fixation or up to days or weeks later. The labelling efficiency decreases, however, with increasing pre-fixation time. A modification of this method is the attachment of a solid crystal of DiI or DiO onto fresh non-fixed tissue, and its subsequent fixation. Irrespective of the fixative or its concentration, the labelled tissue can be stored at $4{ }^{\circ} \mathrm{C}$ or at RT in darkness. The success of labelling can be estimated roughly, and the tissue can be sectioned with a vibratome. Cryosectioning of the tissue is not recommended, since some dye leakage occurs during the procedure.

Although the rate of staining is extremely difficult to assess, estimates range from 100 to $400 \mu \mathrm{m} /$ day. In the example of the visual system, 2 weeks are sufficient to label axons over a distance of $5 \mathrm{~mm}$, and 6 weeks were enough to stain axons over a distance of $12 \mathrm{~mm}$, which is the total length of the retinotectal projection. Since the dye molecules migrate along the plane of the plasma membrane, the average distance they can move over the axon is proportional to the square root of the time period of incubation. In practice, this means that the staining efficacy decreases with increasing distance from the site of application. A further parameter con- trolling the diffusion velocity is the constitution of biological plasma membranes, which differs between species and with embryonic age. The diffusion coefficient of DiI has been estimated to be in the order of magnitude of $10^{-8} \mathrm{~cm}^{2} \mathrm{~s}^{-1}$. Godement et al. (1987) estimated that in the formalin-fixed visual system the diffusion coefficient is $10^{-7} \mathrm{~cm}^{2} \mathrm{~s}^{-1}$ for DiI, which is higher than in living membranes but much lower than the velocity of active axonal transport in vivo. The kinetics of incorporation of DiI molecules in fixed membranes is unknown. The visualisation of DiIlabelled tissue can be performed with conventional fluorescence microscopy, but the dye can also be converted to a substrate that is suitable for EM-level investigation using the DAB fluorescence photoconversion method, as described for LY (Bentivoglio and $\mathrm{Su}$, 1990) and DiAsp (Thanos et al., 1994 for review). Multiple labelling can be achieved by using DiI and $\mathrm{DiO}$ in a single tissue specimen (Godement et al., 1987).

Besides some of the obvious advantages of the postmortem staining methods, most striking are their feasibility for a number of experimental situations. These include wild-living animals, those living under natural protection, human tissue that is only accessible postmortem, and embryonic tissue in utero. Demonstrations of such applications include high-resolution staining of human retinal ganglion cells (Fig. 4A) retrieved from post-mortem eyes up to $48 \mathrm{~h}$ after death (Thanos et al., 1991), and staining of deeper intraretinal horizontal cells (Fig. 4B) and amakrine tissue (Fig. 4C, D), as they can be assessed in tissue that is retrieved during the enucleation of diseased eyes (Janssen et al., 1997).

\subsection{Developmental studies}

The major concepts that forced the introduction of fluorescent probes into developmental studies were those of axonal guidance and, to lesser extent, cell lineage and differentiation. RITC was perhaps the first reported dye to have been applied in vivo (Thanos and Bonhoeffer, 1983). Although not completely lipophilic, it slowly hydrolyses and may be injected locally or applied as a solid crystal. The dye can be transported by itself, or it can bind to transported peptides, therefore enhancing the intensity of the resulting fluorescence. Other dyes with more lipophilic properties are the aforementioned carbocyanines DiI, DiO and DiA, which also have superior properties that make them suitable for the study of developmental systems in vivo (Honig and Hume, 1989; Thanos and Bonhoeffer, 1987; Vanselow et al., 1989). In principle, all dyes that have been reported to be transported or diffuse in adult tissue are also applicable to developing nerves. 
These dyes include the carbocyanines, stilbidin-derivatives, dextran-amines and cyanates.

\subsection{Phagocytosis-dependent staining of glial cells}

Retrograde and anterograde degeneration have been reported to be sufficient stimuli to activate glial cells which, in turn, are involved in the phagocytosis of degenerating material. The phagocytosis-dependent staining of glial cells (Fig. 5) can be used in combination with lectin staining and immunocytochemistry to analyse the involvement of glial cells in the phagocytosis of degenerated axons following a lesion in the CNS, and to correlate phagocytosis with changed glial cell morphology.

Here we describe a double-fluorescence technique
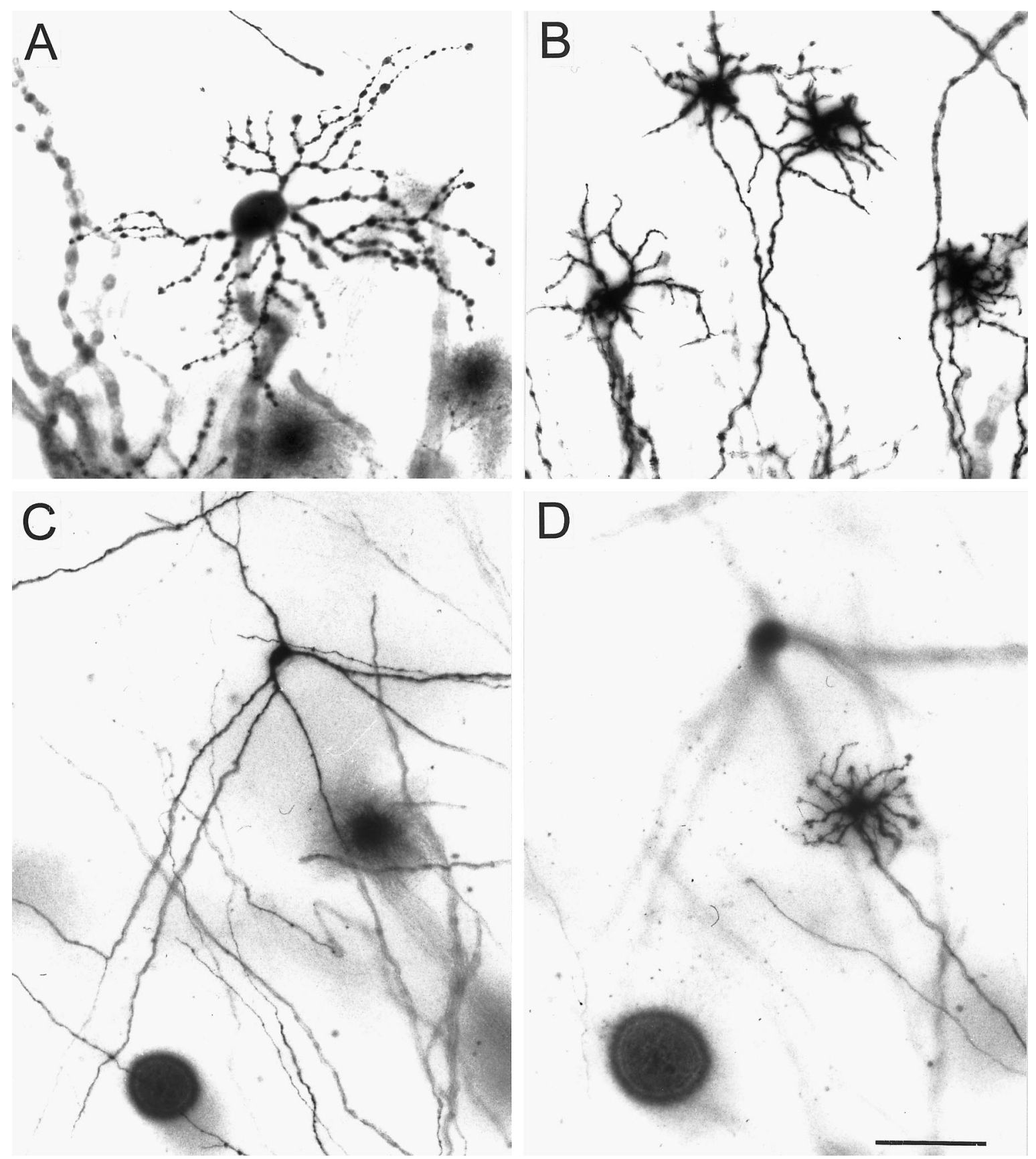

Fig. 4. (A) Human retinal ganglion cell labelled with DiI in a post-mortem retina obtained $48 \mathrm{~h}$ after death. Note the complete staining of the dendrites. (B) Horizontal cells retrogradely stained in a PFA-fixed human retina that is depleted of ganglion cells due to advanced glaucoma. DiI was applied at the telodendrons, and diffusion of the dye occurred over 7 days at $37^{\circ} \mathrm{C}$. (C, D), Staining of cells (focused in C) and a neighbouring horizontal cell (focused in D) photographed in a glaucomatous human eye 7 days after the application of DiI to the inner nuclear layer. Scale bar: $25 \mu \mathrm{m}$. 
that allows for the direct and simultaneous visualisation of both labelled incorporated axonal debris and incorporating glial cells in the course of anterograde degeneration (Bechmann and Nitsch, 1997a, 1997b). The stereotaxic application of small crystals of biotinylated and tetramethylrhodamine isothiocyanate (TRITC)-conjugated dextran amine MR into the medial entorhinal cortex (EC) has been shown to result in a stable rhodamine fluorescence that is confined to the fibres and terminals in the middle molecular layer of the dentate gyrus, the stratum lacunosum-moleculare, and the crossed temporo-hippocampal pathway. A subsequent stereotaxic lesion of the EC induced the transformation of rhodamine-fluorescent fibres and terminals into small granules. The incorporation of these granules into microglial cells, labelled by FITCcoupled isolectin B4 of Bandeiraea simplicifolia (BSI$\mathrm{B}_{4}$ ), or astrocytes, labelled by FITC-conjugated glial fibrillary acidic protein (GFAP) antibodies, resulted in the phagocytosis-dependent labelling of these nonneuronal cells, which could be identified by double-fluorescence microscopy (see Bechmann and Nitsch, 1997a, 1997b).

Stereotaxic surgery was performed using adult rats (290-310 g) under deep Nembutal anaesthesia $(50 \mathrm{mg} /$ $\mathrm{kg}$ body weight). Both tracer application and lesion were performed solely in the left hemisphere of the brains. The tracer was applied using a conventional spinal needle consisting of an inner needle ensheathed by a metal envelope. The most distal $2.5 \mathrm{~mm}$ of this envelope was cut to allow the inner needle to contact only the entorhinal region (layers II and III), but not brain areas above the EC and the meninges. By doing this, labelling of the meningeal macrophages and brain regions other than the target area could be minimised. After stereotaxic placement of the envelope into the correct position (anterior-posterior (AP) +0.3, lateral (L) 4.0-5.5, and vertical (V) -4.1 as measured from lambda (Paxinos and Watson, 1986)), the tip of the inner needle was soaked with $0.9 \% \mathrm{NaCl}$, brought into contact with only few crystals of MR, and guided within the envelope into the EC. Since the needle was exposed for $2.5 \mathrm{~mm}$, the entorhinal area was labelled from $\mathrm{V} 4.1$ to 6.6. One day following tracer application, the rats received an electrolytic EC lesion, as described in detail by Deller et al., (1995). Animals in which an electrolytic EC lesion was not performed were used as controls for the fading of the tracer, lectin and immunostaining, and were perfused transcardially either 1 or 20 days after the application of MR. The lesioned animals were anaesthetised and perfusion-fixed from $12 \mathrm{~h}$ to 20 days after lesioning, and their brains were sectioned on a vibratome (details in Deller et al., 1995). For double-fluorescence light and confocal microscopy, microglial cells were stained using FITC-conjugated BSI-B4 (Sigma L-2895; Streit and Kreutzberg, 1987). Sections were incubated overnight in the dark at $4^{\circ} \mathrm{C}$ in $20 \mu \mathrm{g}$ BSI-B4/ml containing

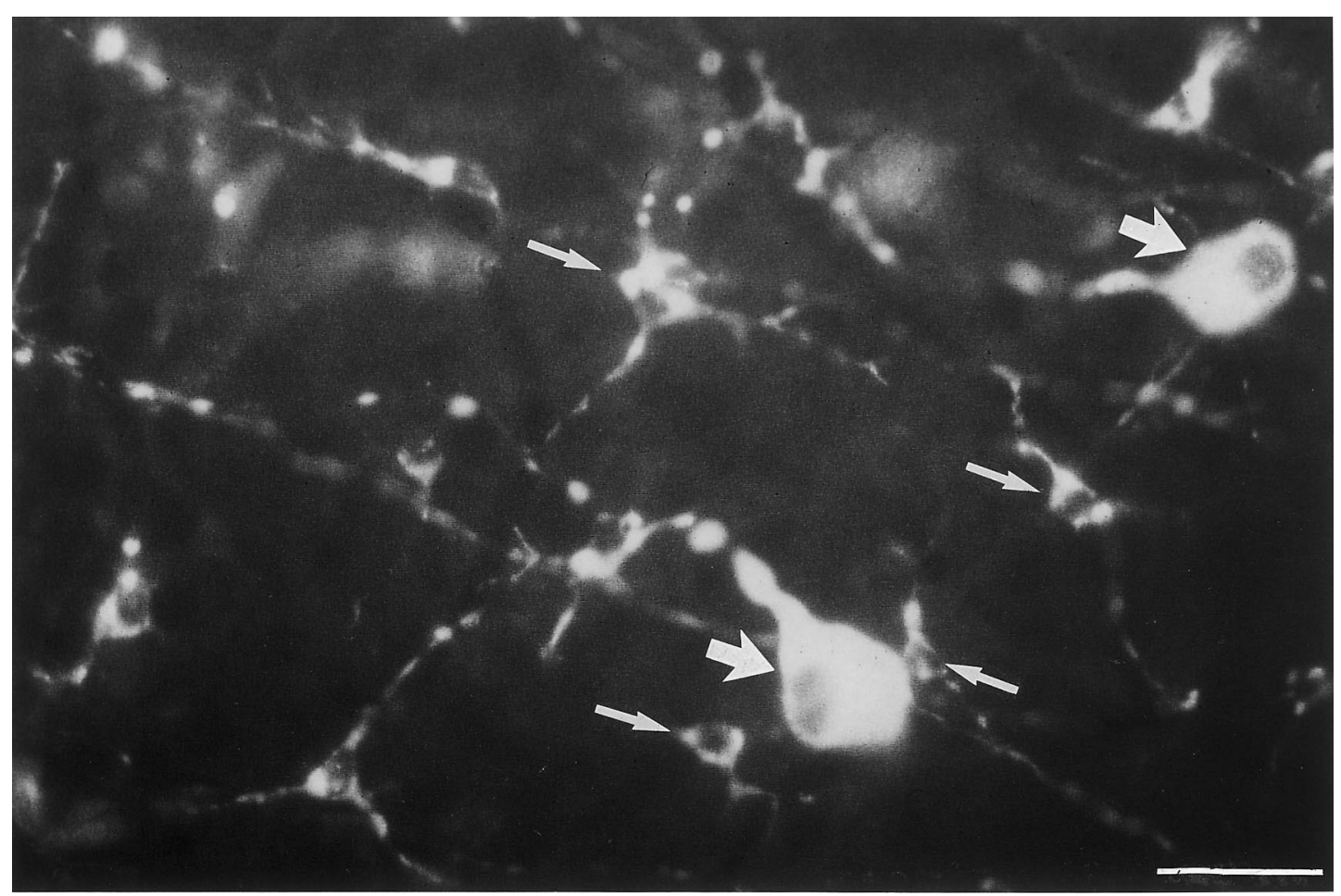

Fig. 5. Degeneration of prelabelled retinal ganglion cells (RGCs) results in a progressive phagocytosis-dependent staining of microglial cells (small arrow), while RGCs that are still alive are also visible (big arrow). Scale bar: $10 \mu \mathrm{m}$. 
$0.1 \%$ Triton X-100. After several washes in PBS,sections were mounted and coverslipped. Astroglia were stained by incubation in a rabbit primary antibody raised against GFAP (diluted 1:500 in PBS containing $0.5 \%$ Triton X-100 and 2\% normal sheep serum),overnight at $4{ }^{\circ} \mathrm{C}$. Sections were then immersed in a sheep anti-rabbit IgG-FITC secondary antibody (diluted 1:20 in PBS) for $4 \mathrm{~h}$. Sections were washed thoroughly, mounted, and coverslipped in Immomount. Fluorescence was observed using different FITC, TRITC and combined FITC/TRITC filter sets.

The application of small crystals of MR into the medial EC using conventional spinal needles led to a fast (day 1), strong and stable staining of the known efferent connection of the EC (Witter, 1993), but not to transcellular staining, even after long survival times (20 days). This is an important feature of MR, since transcellular travelling would open up the possibility that glial labelling cannot be interpreted only as phagocytosis of labelled debris, but also as the result of leakage, as has been reported for FG (McBride et al., 1988). Dextran amines are highly hydrophilic molecules that must be taken up actively and diffuse throughout the cytoplasm (Fritzsch and Sonntag, 1991; Ohm and Dieckmann, 1994). Once inside a cell, they cannot cross the membrane. The data from these experiments confirm this hypothesis, since the rhodamine signal remained restricted to fibres and terminals, and did not diffuse into the neuropil, even after long survival times. The application results in an inevitable spreading of the tracer along the perivascular spaces, where it is phagocytosed by perivascular cells (Mato et al., 1985; Streit and Graeber, 1993). This results in a free diffusion of the hydrophilic dextran amine within the cytoplasm, which leads to staining of the entire cell, as can be observed in the labelled neurones of the contralateral EC. In contrast, glial cells (identified by lectin staining or immunocytochemistry using doublefluorescence light and confocal microscopy) were not labelled homogeneously, but exhibited a rather granular staining, which reflects the ultrastructural finding that the tracer does not leak out of degenerating neurones. Taken together, it must be concluded that the intracellular granules around the nucleus do, in fact, represent pre-labelled degenerated axons containing the tracer, which have been incorporated by phagocytosis.

\subsection{Electrophysiological mapping combined with neural tract tracing}

A feature common to many investigations of the CNS is a desire to chart the anatomical connections of different functionally-identified regions. The study of the cerebellum is no exception and a key organisational feature of its cortex is a division into a number of longitudinal strips or zones, each about $1 \mathrm{~mm}$ in mediolateral width but extending over many folia in the rostrocaudal axis (Armstrong, 1990; Oscarsson, 1980; Voogd and Ruigrok, 1997). However, the location of these zones varies somewhat between animals
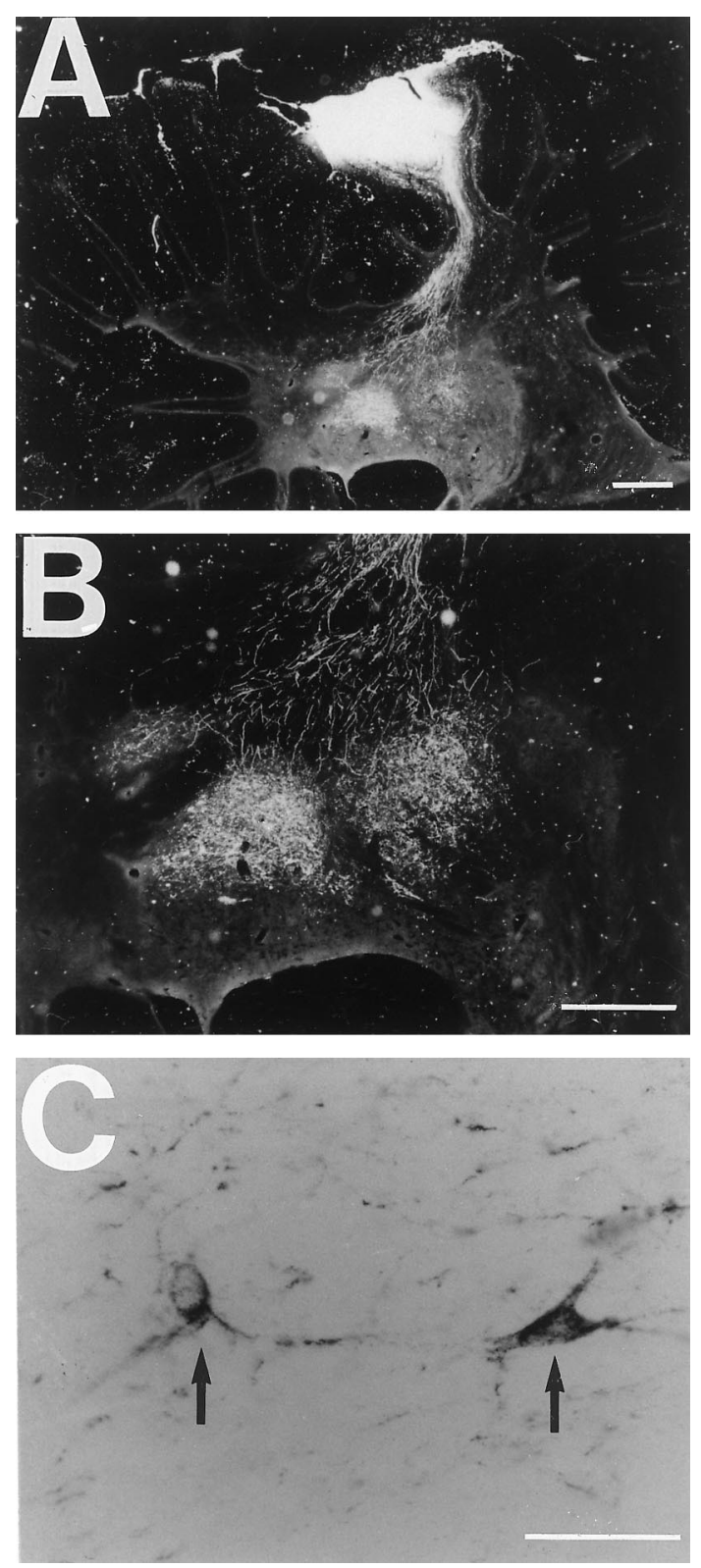

Fig. 6. Photomicrographs illustrating (A) the appearance of a wheatgerm-agglutinin-conjugated horseradish peroxidase (WGA-HRP) injection site in the cerebellar cortex (lobule $\mathrm{Vb} / \mathrm{c}$ ) viewed with the aid of dark-field illumination and using partially crossed polarising filters. Note also the tract of labelled axons and terminal labelling within the nucleus interpositus. (B) Higher power, dark-field view of the same section as in A to show anterograde terminal labelling in both divisions of nucleus interpositus (heavier in interpositus posterior). (C) Higher power bright-field view of the section depicted in $\mathrm{B}$, to show examples of retrogradely labelled cells (arrows) in interpositus posterior. Scale bars: A, $1 \mathrm{~mm}$; B and C, $100 \mu \mathrm{m}$. (Modified from Trott et al., 1990). 
and they are not visible on the cortical surface. It is therefore necessary to use electrophysiological mapping techniques to guide the placement of neural tracer material into a selected cortical zone.

The aim of the present section is to outline a series of studies that have combined electrophysiological methods with the use of three different classes of axonal tracers to chart cerebellar input and output pathways. The tracers in question are WGA-HRP, fluorescent-tagged microspheres (beads) and the dextran amine FR and the present section highlights some of these tracers' strengths and weaknesses, as revealed by such experiments. As the methodological details of the electrophysiology are presented in a number of previous papers (Trott et al., 1990; Trott et al., 1998a, 1998b; Apps and Garwicz, 2000) they will not be repeated here.

\subsubsection{WGA-HRP experiments and electrophysiology}

Gonatas et al. (1979) were the first to describe the use of HRP conjugated to the lectin WGA as an axonal tracer with a sensitivity 40 times greater than that of free HRP. Both tracers are taken up by damaged fibres of passage but, unlike free HRP, WGA-HRP is not thought to be taken up by intact fibres passing through the injection site (Brodal et al., 1983). As a result of these and other advantages (Mesulam, 1982), WGA-HRP has become one of the most widely used neuroanatomical tracers. Its popularity remains undiminished, not only for studies of CNS connections at the light-microscope (LM) level, but also in studies of ultrastructural detail (Teune et al., 1998). It has also proven to be an effective transneuronal tracer, permitting studies of multisynaptic pathways (Krug et al., 1998). However, given that its application in studies of neural connections has been reviewed comprehensively (Bolam, 1992; Van der Want et al., 1997), consideration here is confined to its use in LM studies of the connections of cerebellar cortical zones.

WGA-HRP is a highly sensitive bi-directional tracer, resulting in the anterograde labelling of axon terminals and retrograde labelling of cell bodies alike (Fig. 6). As in studies of other CNS pathways it has been used extensively to chart the connections of the mammalian cerebellum, including some of the most recent investigations of the input and output pathways associated with different cerebellar cortical zones (Apps and Trott, 1998; Trott et al., 1998a, 1998b). In such studies the locations and mediolateral widths of the cortical zones are first mapped using electrophysiological techniques and the mapping is then used to guide the placement of a single, small (10-30 nl) injection of $1-2 \%$ WGA-HRP approximately $0.5 \mathrm{~mm}$ below the cerebellar surface, into the centre of a chosen zone. Injections are made hydraulically via a glass micropipette (tip diameter $10-20 \mu \mathrm{m}$ ) attached to a 1 $\mu 1$ microsyringe. Since the WGA-HRP solution is almost colourless, a small quantity of Luxol fast blue is sometimes added to the tracer solution to aid visualisation of the injectate. The micropipette is left in situ for 1-2 min and, after its removal, the brain surface is thoroughly rinsed with sterile saline before the wound is closed in layers.

WGA-HRP is known to be transported rapidly (anterograde transport rates are thought to exceed 300 $\mathrm{mm} /$ day, while retrograde rates are in the order of 100 mm/day, see Mesulam, 1982; Gibson et al., 1984 for details). It is therefore likely that the overall transport rate is influenced heavily by other factors, including uptake time and choice of anaesthetic. Pentobarbitone, for example, is known to inhibit the transport of HRP (Mesulam and Mufson, 1980), and, in our hands, a survival time of less than 2 days fails to produce detectable retrograde labelling in the inferior olive after injection of WGA-HRP into the cerebellar cortex (Trott and Apps, 1993). By contrast, periods in excess of 5 days are considered likely to result in transneuronal labelling and an inaccurate estimation of the size of the effective injection site, as well as some decrease in labelling (Gibson et al., 1984; Mesulam, 1982). A survival period of between 3 and 5 days has therefore been used in studies of cortico-nuclear and olivo-cerebellar connections in the cat (Trott et al., 1998a, 1998b), while $30-40 \mathrm{~h}$ is sufficient for similar studies in rat (Apps, 1990).

\subsubsection{WGA-HRP histochemistry and microscopy}

In all experiments described here, the brainstem and cerebellum are removed after intracardial perfusion and stored as blocks overnight in the refrigerator in $30 \%$ sucrose solution (cats) or $10 \%$ sucrose solution (rats). The following day, frozen sections are cut from the blocks (usually at $50 \mu \mathrm{m}$ thickness) and two series are collected and mounted (via a $1 \%$ gelatin/0.1\% chrome alum solution) directly onto subbed slides, which are allowed to air dry before further processing. Where the sections are particularly fragile, as is the case for the cerebellar sections, and if the tissue is not well fixed, it is advisable to block the tissue in $10 \%$ gelatin prior to cutting. One of the slide-mounted series of sections from each block is then counterstained with neutral red or cresyl violet, and the second series is processed histochemically for HRP using the sensitive chromogen TMB (Mesulam, 1982, Van der Want et al., 1997). Generally, it is advisable to carry out the cutting and processing on the same day, otherwise a significant reduction in enzyme activity can occur. Also, if artefact is a problem, a modified TMB reaction protocol can be used to produce cleaner results (Gibson et al., 1984). 


\subsubsection{Interpretation of the injection site}

One potential limitation of WGA-HRP as a neuronal tracer is the difficulty of interpreting the size of the "effective" injection site, that is the region from which the uptake and subsequent transport of tracer material occurs (Mesulam, 1982). WGA-HRP is highly diffusible and even the smallest hydraulic injection (ca. $10 \mathrm{nl}$ ) can result in quite extensive cortical staining ( $>1 \mathrm{~mm}$ in width). However, the cerebellar cortex and its connections are well suited for evaluation of the effective injection site because the olivocerebellar projection is entirely crossed and therefore the size of injections made near the cerebellar midline can be compared to the extent of bilateral olivary labelling. This makes it possible to demonstrate that a substantial part of the total area of cortical staining (the injection site "halo") does not give rise to detectable olivary labelling (Apps, 1990).

To summarise, WGA-HRP is a highly efficient, bidirectional tracer that continues to provide valuable information regarding central pathways, including the connections of the cerebellar cortex. It does, however, have a number of disadvantages, which include the need for fairly time-consuming histochemistry, the difficulty of producing artefact-free results, and the generation of relatively large injection sites $(>1 \mathrm{~mm}$ in diameter). The smallest size of effective injection site obtained with WGA-HRP is particularly relevant to studies of the cerebellum because this is usually larger than the width of individual cerebellar cortical zones. The patterns of localisation that emerge from such injections are therefore limited in their interpretation.

\subsubsection{Fluorescently-tagged latex microsphere experiments}

Fluorescently-tagged latex microspheres or "beads" were first introduced as a retrograde tracer by Katz et al. (1984). They have the advantage over WGA-HRP and other "conventional" fluorescent tracers that they can produce, at the site of injection, a well-defined and highly restricted region of retrograde uptake. Thus, with regard to the cerebellum, injections can be restricted to within the boundaries of a chosen cortical zone. Both red and green beads are commercially available, permitting the performance of double-labelling studies with tracers that have very similar transport characteristics (in our studies of cerebellar connections best results have been obtained from beads supplied by Lumafluor Inc, New City, NY, USA). The beads have also been used to carry active agents to produce selective destruction of subpopulations of retrogradely labelled cells (Hafeli et al., 1995; Madison et al., 1990).

Like other fluorescent tracers, the results obtained using beads are non-permanent. Once sectioned, the material is best inspected fresh (ideally within 1 or 2 weeks) and any photomicrographs should be taken as soon as possible (in our hands the best results have been obtained at $\times 40$ magnification using a 200 ASA Agfachrome colour slide film, Apps, 2000). Levels of autofluorescence in the sections increase progressively over time so that eventually even the brightest labelling is obscured. However, the tissue can be stored as blocks in sucrose solution in the refrigerator for long periods before sectioning, without any apparent loss of fluorescent labelling.

Cells are retrogradely labelled with red or green beads at a wide range of intensities, but are readily differentiated from background by the granular appearance of their cytoplasm (Apps, 1998; Apps, 2000 Apps et al., 1991; King et al., 1998). Cells brightly labelled with red beads can, however, interfere with the identification of cells that are more weakly labelled with green beads. To overcome this problem, a low-pass $(560 \mathrm{~nm})$ filter can be incorporated into the light path to reduce the red fluorescent "shine through".

\subsubsection{Efficiency of the red and green beads as retrograde tracers}

For double-labelling studies it is important to assess the extent to which the two different tracers used are equally efficient at producing detectable retrograde cell labelling. Control experiments have therefore been carried out in which the red and green beads have been mixed together in equal proportions. Following injections of the mixture into the cerebellar cortex, every olive cell that was retrogradely labelled with one colour of beads was also found to be labelled with the other (Apps and Garwicz, 2000; Katz and Iarovici, 1990), indicating that the two tracers are taken up and transported with equal efficiency.

The "effective" injection site from which the uptake and retrograde transport of tracer material occurs is also an important factor when considering the findings from neural tract-tracing experiments. A common feature of fluorescent tracers is that the injection site can usually be divided into a central, intensely labelled core, surrounded by a weakly labelled halo. With beads the core (as with many other tracers) is normally considered to be the region of uptake. However, by contrast to other fluorescent tracers (including for example, FB, FG, and DY), the transition from core to halo is rather abrupt, facilitating the measurement of a discrete injection site (Apps et al., 1991). Quantitative studies have also shown that no statistically significant correlation is present between the numbers of cells retrogradely labelled with beads and the volume of injectate (King et al., 1998). This is a finding that is not altogether surprising, given that the presence of an intensely fluorescent injection site implies that the amount of tracer injected is much more than can be 
removed by axonal transport. On the other hand, there is evidence to suggest that the size of the injection site influences the number of cells that are retrogradely labelled with beads (King et al., 1998). Again this is as expected, although it is noteworthy that the correlations were not particularly high (at best $54 \%$ of the observed variation in numbers of retrogradely labelled cells was attributable to the variation in injection site size), implying inaccuracies of injection site measurement and/or cell counts. Nevertheless, any such errors in measurement are not likely to be of sufficient magnitude to alter the conclusion that the beads are highly efficient retrograde tracers.

To summarise, beads are well suited for retrograde tracing studies in which small injection sites are sought $(<1 \mathrm{~mm}$ in size), including studies of the zonal organisation of the cerebellar cortex. They are very reliable, extremely efficient, simple to use and permit doublelabelling studies of axonal branching. The retrograde labelling is straightforward to identify, and both the red and the green fluorescence are resistant to photobleaching. However, the beads are transported exclusively in the retrograde direction and have only limited compatibility with other histochemical techniques. Procedures that include ethanol, for example, will cause serious fading of the labelling.

\subsubsection{Fluoro-Ruby experiments}

FR, often used as a $15-20 \%$ solution in water, was introduced as a new class of bi-directional tracer by Schmued et al., 1990 (Nance and Burns, 1990). FR and its derivative, biotinylated dextran amine (BDA, Brandt and Apkarian, 1992; Rajakumar et al., 1993; Veenman et al., 1992) have proven to be valuable new tracers in studies of central connections.

Excellent results in both rat and cat have been obtained with FR, particularly in tracing anterograde pathways, and in studies of cerebellar cortico-nuclear connections it has been successfully used for investigations of the fine-grain topographical organisation of individual cortical zones (e.g., Garwicz et al., 1996; Apps and Garwicz, 2000). By contrast, results have been disappointing in similar experiments (at least in cats) in which BDA has been used, although it is very effective at labelling afferent pathways to the inferior olive (Apps, 1998). The fluorescence from FR is viewed 5-15 days after injection, using the same rhodamine filter block as described above for the red beads.

FR labelling is resistant to photobleaching and does not seem to leak out from labelled processes. An additional advantage is the tracer's compatibility with other histochemical techniques, including HRP histochemistry (Schmued et al., 1990). If required, FR can also be photo-converted (Schmued and Snavely, 1993) or labelled with a primary antibody (Chang et al.,
1990; Kaneko et al., 1996) to produce an electrondense product that is suitable for ultrastructural analysis. As with most other tracers it is likely to be taken up by damaged fibres of passage (Nance and Burns, 1990). Its high sensitivity can also result in collateralcollateral labelling. That is, retrograde transport of the tracer from afferent terminals at the injection site back to a branch point in the stem axon, and then anterograde transport to other terminal branches of the same neurone (Apps, unpublished observations). In fact, this phenomenon is likely to be a characteristic of most, if not all bi-directional tracers (Chen and Aston-Jones, 1998; Mesulam, 1982), and care must be taken to ensure that any such indirect labelling is not misinterpreted as anterograde transport arising directly from cell bodies at the injection site.

In summary, FR is a highly sensitive anterograde tracer that can be used to produce small injection sites $(<1 \mathrm{~mm}$ in width) to map fine-grain topographical connections within the CNS. Its compatibility with other methods, ease of use and high resistance to fading make it an ideal fluorescent tracer. One disadvantage, however, is its transport in the retrograde direction, which is inferior to that of other fluorescent tracers, most notably the fluorescently-tagged beads.

\subsection{Multiple-labelling experiments}

The use of animals in neuroscience is under continuous and increasingly critical observation by society at large. In multiple-tracing experiments a marked reduction in the use of experimental animals is anticipated, while simultaneously more scientific data about the organisation of the brain are obtained. Using three different tracers in the same animal, the added value is that not only can three projection areas be established while reducing the number of animals used by twothirds, but also that it is possible to delineate accurately the degree of convergence or divergence of the involved projections.

\subsubsection{The triple staining procedure}

Two issues have played key roles in the achievement of the triple-staining described here. Firstly, a recent report (Zhou and Grofova, 1995) concerning the use of the peroxidase substrate Vector ${ }^{\circledR}$ VIP (V-VIP) as an alternative to DAB has allowed the design of a procedure enabling the simultaneous and permanent visualisation of three markers according to a three-colour paradigm (Lanciego et al., 1997). Secondly, in preparations for EM the V-VIP coloured product exists as a dense, granular precipitate that can be distinguished from the flocculent DAB precipitate. The introduction of a highly specific antiserum against FG raised in rabbit (Chang et al., 1990; Van Bockstaele et al., 1994), has enabled the improved detection of FG via immu- 
noperoxidase techniques, and therefore renders other currently available detection procedures unnecessary, such as photoconversion under UV light (Balercia et al., 1992; Bentivoglio and Su, 1990). Finally, the immunocytochemical visualisation of FG facilitates the combination of this retrograde tracer with other markers (Fig. 7).

Briefly, the first tracer is visualised histochemically with an $A B C$ solution and is finally stained with the chromogen nickel-enhanced DAB (DAB-Ni), resulting in a blue-black precipitate. The second and third markers are detected by means of two immunoperoxidase techniques running simultaneously as far as the incubations with antisera are concerned, and then stained by using $\mathrm{DAB}$ as the second chromogen (brown reaction product) and then V-VIP for the visualisation of the third marker (purple reaction product) (Fig. 7).
After the triple-staining, it is possible to study the degree of overlap of a particular projection input onto two individual subsets of projection neurones (Lanciego et al., 1998a; multiple axonal tracing combining anterograde transport of BDA with dual retrograde axonal transport of $\mathrm{CTB}$ and FG). It is also possible to study the degree of convergence or divergence that exists between two different terminal fields of projections within a population of retrogradely labelled neurones (Lanciego et al., 1998b; dual anterograde axonal tracing with BDA and PHA-L in combination with retrograde tracing using $\mathrm{FG}$ ). Another natural choice (Lanciego et al., 1997) is the simultaneous detection of anterogradely transported BDA, retrogradely transported FG and the immunohistochemical detection of markers like the calcium-binding proteins calbindin and parvalbumin.

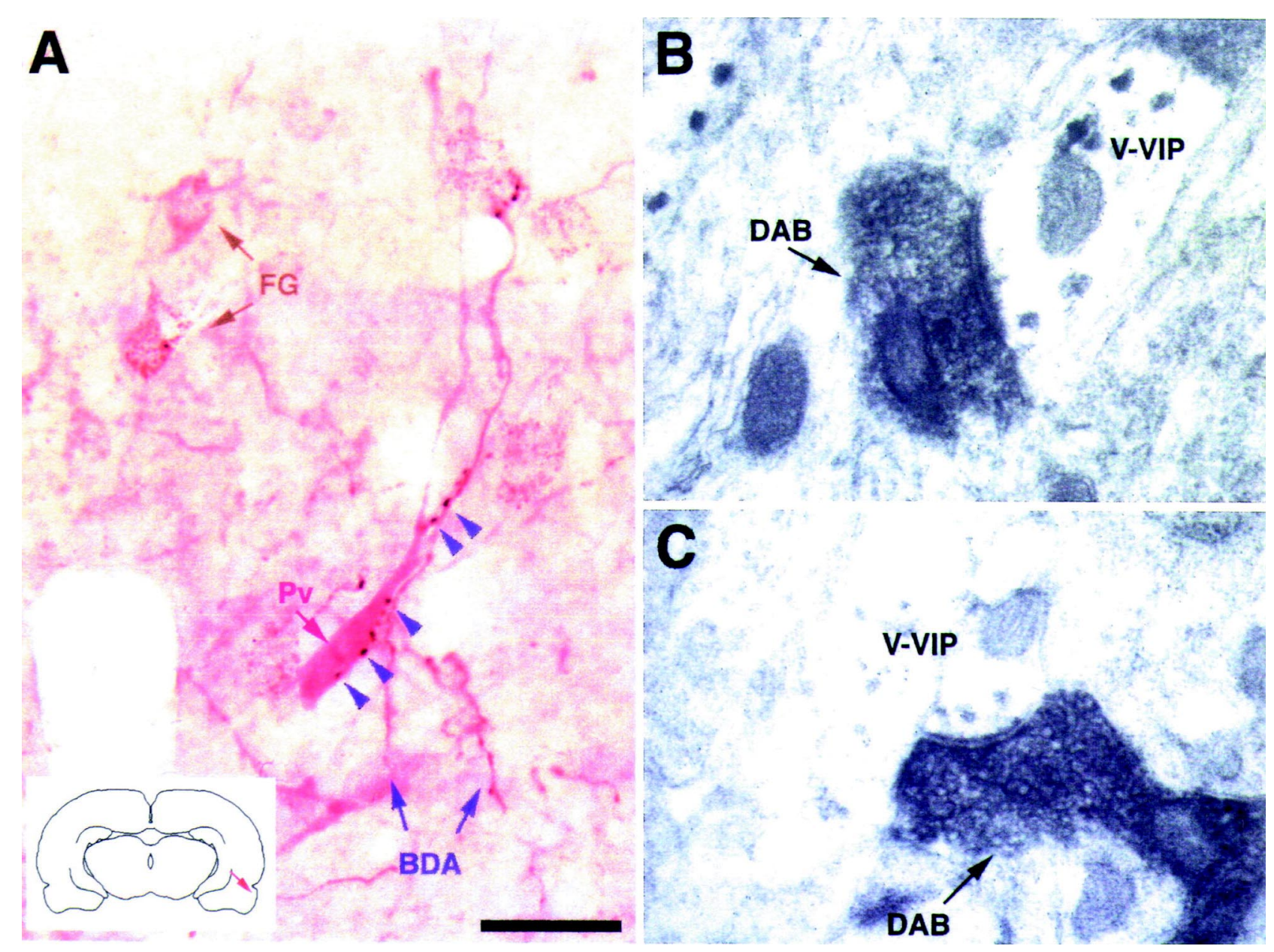

Fig. 7. Use of dual tract-tracing protocols in combination with the detection of neuroactive substances. (A) Superficial layers of the rat entorhinal cortex. The three-colour paradigm is used for the simultaneous detection of afferents from the perirhinal cortex (anterograde tracing with biotinylated dextran amine - BDA, black fibres, blue arrows), principal layer II cells projecting to the hippocampus (retrograde tracing with FG, brown granular cells, brown arrows), and a subpopulation of layer III interneurones (parvalbumin-immunoreactive neurones, purple-stained, see purple arrow). Blue arrowheads show presumptive contacts between BDA-labelled axons and parvalbumin-immunoreactive neurones. Scale bar: $35 \mu \mathrm{m}$. (B and C) Fine morphology of the superficial entorhinal layers. A double pre-embedding procedure was carried out to visualise perirhinal afferents (BDA-labelled, diaminobenzidine reaction product, black flocculent precipitate) as well as parvalbumin-immunoreactive profiles (V-VIP; dense granular reaction product). Note that the typical electron-dense granular deposit belongs to the V-VIP peroxidase substrate. 


\subsubsection{Detailed histological procedure}

In order to ensure sufficient detail for the protocol to be replicated, an extensive, step-by-step description of the triple-staining methods used is given below. The combination of BDA $+\mathrm{CTB}+\mathrm{FG}$ is taken as an example. Additional information regarding tracer delivery, survival time, perfusion, fixation and sectioning can be obtained elsewhere (Lanciego et al., 1997, 1998a, b). The histological processing of sections is as follows:

- Rinse $3 \times 10 \mathrm{~min}$ in $0.05 \mathrm{M}$ Tris-buffered saline (TBS)-Triton-X pH 8.0, RT.

- Incubate in ABC solution (Vector Labs, 90 min, RT).

- Rinse $2 \times 10 \mathrm{~min}$ in $0.05 \mathrm{M}$ TBS-Tx pH 8.0 (RT).

- Rinse $2 \times 10 \mathrm{~min}$ in $0.05 \mathrm{M}$ Tris $/ \mathrm{HCl} \mathrm{pH} 8.0$ (RT).

- Incubate in DAB-Ni (5 min, RT). DAB-Ni solution is prepared as follows: $200 \mathrm{mg}$ of nickel ammonium sulphate in $50 \mathrm{ml}$ of Tris $/ \mathrm{HCl} \mathrm{pH} \mathrm{8.0.} \mathrm{Once} \mathrm{dis-}$ solved, add $7.5 \mathrm{mg}$ of DAB and then $10 \mu \mathrm{l}$ of $\mathrm{H}_{2} \mathrm{O}_{2}$.

- Rinse $3 \times 10 \mathrm{~min}$ in $0.05 \mathrm{M}$ Tris $/ \mathrm{HCl} \mathrm{pH} 8.0(\mathrm{RT})$.

- Rinse $3 \times 10$ min in 0.05 M TBS-Tx pH 8.0 (RT).

- Incubation in a cocktail solution of primary antisera comprising 1:2000 goat anti-CTB (List Biological) and 1:2000 rabbit anti-FG (Chemicon) in $0.05 \mathrm{M}$ TBS-Tx pH $8.0+2 \%$ bovine serum albumin $(60 \mathrm{~h}$, $\left.4^{\circ} \mathrm{C}\right)$.

- Rinse $3 \times 10 \mathrm{~min}$ in $0.05 \mathrm{M}$ TBS-Tx pH 8.0 (RT).

- Incubate in a cocktail solution of secondary antisera containing 1:50 donkey anti-goat IgG (Nordic or Sigma) and 1:50 swine anti-rabbit IgG (Dakopatts) in $0.05 \mathrm{M}$ TBS-Tx pH 8.0 ( $2 \mathrm{~h}, \mathrm{RT})$.

- Rinse $3 \times 10$ min in 0.05 M TBS-Tx pH 8.0 (RT).

- Incubate in a PAP complex raised in goat (Nordic or Sigma). 1:600 goat-PAP (90 min, RT).

- Rinse $2 \times 10 \mathrm{~min}$ in $0.05 \mathrm{M}$ TBS-Tx pH 8.0 (RT).

- Rinse $1 \times 10 \mathrm{~min}$ in $0.05 \mathrm{M}$ Tris $/ \mathrm{HCl} \mathrm{pH} 8.0(\mathrm{RT})$.

- Rinse $2 \times 10 \mathrm{~min}$ in $0.05 \mathrm{M}$ Tris $/ \mathrm{HCl} \mathrm{pH} 7.6(\mathrm{RT})$.

- Incubate in a DAB solution (20-30 min, RT). A regular $\mathrm{DAB}$ solution is prepared by dissolving 5 $\mathrm{mg}$ of $\mathrm{DAB}$ in $10 \mathrm{ml}$ of $0.05 \mathrm{M}$ Tris $/ \mathrm{HCl} \mathrm{pH} \mathrm{7.6.}$ Once dissolved, add $3.3 \mu$ l of $\mathrm{H}_{2} \mathrm{O}_{2}$.

- Rinse $3 \times 10 \mathrm{~min}$ in $0.05 \mathrm{M}$ Tris $/ \mathrm{HCl} \mathrm{pH} 7.6(\mathrm{RT})$.

- Rinse $1 \times 10 \mathrm{~min}$ in $0.05 \mathrm{M}$ Tris $/ \mathrm{HCl} \mathrm{pH} 8.0(\mathrm{RT})$.

- Rinse $2 \times 10 \mathrm{~min}$ in $0.05 \mathrm{M}$ TBS-Tx pH 8.0 (RT).

- Incubate in a PAP complex raised in rabbit (Dakopatts). 1:600 rabbit-PAP (90 min, RT).

- Rinse $2 \times 10$ min in 0.05 M TBS-Tx pH 8.0 (RT).

- Rinse $1 \times 10 \mathrm{~min}$ in $0.05 \mathrm{M}$ Tris $/ \mathrm{HCl} \mathrm{pH} 8.0(\mathrm{RT})$.

- Rinse $2 \times 10 \mathrm{~min}$ in $0.05 \mathrm{M}$ Tris $/ \mathrm{HCl}$ pH $7.6(\mathrm{RT})$.

- Incubate in the V-VIP peroxidase substrate (Vector). V-VIP solution is prepared by adding 1 drop of each vial (the V-VIP substrate is presented as a kit containing 4 vials) to $3.5 \mathrm{ml}$ of $0.05 \mathrm{M}$ Tris $/ \mathrm{HCl} \mathrm{pH}$
7.6 (5-10 min, RT).

- Rinse $3 \times 10 \mathrm{~min}$ in $0.05 \mathrm{M}$ Tris $/ \mathrm{HCl} \mathrm{pH} 7.6(\mathrm{RT})$.

\section{Perspectives}

There is little doubt that modern tract-tracing protocols will continue to be very useful in basic neuroanatomical research. The further study of the complexity of brain circuits requires the use of powerful multi-labelling procedures that are aimed at visualising simultaneously several projections, because single- or even double-staining approaches may often not provide enough detail. The versatility of the three-colour paradigm described in the present report might be of considerable use to neuroanatomists, while reducing the number of test animals required. Moreover, this procedure has some advantages over the fluorescent markers that are nowadays commonly used in multiple neuroanatomical tract-tracing methods, for example the stability of the staining and compatibility with EM.

\section{References}

Akintunde, A., Buxton, D.F., 1992. Quadruple labeling of brain-system neurones: a multiple retrograde fluorescent tracer study of axonal collateralization. J. Neurosci. Methods 45, 15-22.

Apps, R., 1990. Columnar organisation of the inferior olive projection to the posterior lobe of the rat cerebellum. J. Comp. Neurol. 302, 236-254.

Apps, R., Trott, J.R., Dietrich, E., 1991. A study of branching in the projection from the inferior olive to the $\mathrm{x}$-zone and lateral $\mathrm{cl}$ zone of the cat cerebellum using a combined electrophysiological and retrograde fluorescent double-labelling technique. Exp. Brain Res. 87, 141-152.

Apps, R., 1998. Input-output connections of the "hindlimb" region of the inferior olive in cats. J. Comp. Neurol. 399, 513-529.

Apps, R., Trott, J.R., 1998. Topographical organisation within the lateral reticular nucleus mossy fibre projections to the $c_{1}$ and $c_{2}$ zones in the rostral paramedian lobule of the cat cerebellum. J. Comp. Neurol. 381, 175-187.

Apps, R., 2000. Rostrocaudal branching within the climbing fibre projection to forelimb-receiving areas of the cerebellar cortical $\mathrm{C} 1$ zone. J. Comp. Neurol. 419, 193-204.

Apps, R., Garwicz, M., 2000. Precise matching of C1-C3 zone olivocortical divergence and cortico-nuclear convergence in the paravermal cerebellum. Eur. J. Neurosci. 12, 205-214.

Armstrong, D.M., 1990. Topographical localisation in the projections from the inferior olive to the paravermal cortex of the anterior lobe and paramedian lobule in the cerebellum of the cat. Arch. Ital. Biol. 128, 183-207.

Balercia, G., Chen, S., Bentivoglio, M., 1992. Electron microscopic analysis of fluorescent neuronal labeling after photoconversion. J. Neurosci. Methods 45, 87-98.

Bechmann, I., Nitsch, R., 1997a. Astrocytes and microglial cells incorporate degenerating fibres following entorhinal lesion: a light, confocal, and electron microscopical study using a phagocytosisdependent labelling technique. Glia 20, 145-154. 
Bechmann, I., Nitsch, R., 1997b. Identification of phagocytic glial cells after lesion-induced anterograde degeneration using doublefluorescence labelling: combination of axonal tracing and lectin or immunostaining. Histochem. Cell Biol. 107, 391-397.

Bentivoglio, M., Kuypers, H.G., Catsman-Berrevoets, C.E., Loewe, H., Dann, O., 1980. Two new fluorescent retrograde neuronal tracers which are transported over long distances. Neurosci. Lett. 18, 25-30.

Bentivoglio, M., Su, H.S., 1990. Photoconversion of fluorescent retrograde tracers. Neurosci. Lett. 113, 127-133.

Björklund, A., Skagerberg, G., 1979. Simultaneous use of retrograde fluorescent tracers and fluorescence histochemistry for convenient and precise mapping of monoaminergic projections and collateral arrangements in the CNS. J. Neurosci. Methods 1, 261-277.

Bolam, J.P., 1992. Experimental neuroanatomy. A practical approach. In: Rickwood, D, Hames, B.D. (Eds.), The Practical Approach Series, vol. 114. IRL Press, Oxford University Press, Oxford, pp. 1-273.

Bonhoeffer, F., Huf, J., 1980. Position-dependent properties of retinal axons and their growth-cones. Nature 315, 409-410.

Brandt, H.M., Apkarian, A.V., 1992. Biotin-dextran: a sensitive anterograde tracer for neuroanatomic studies in rat and monkey. J. Neurosci. Methods 45, 35-40.

Brodal, P., Dietrichs, E., Bjaalie, J.G., Nordby, T., Walberg, F., 1983. Is lectin-coupled horseradish peroxidase taken up and transported by undamaged as well as damaged fibres in the central nervous system? Brain Res. 278, 1-9.

Cabot, J.B., Mennone, A., Bogan, N., Carroll, J., Evinger, C., Erichsen, J.T., 1991. Retrograde, trans-synaptic and transneuronal transport of fragment $\mathrm{C}$ of tetanus toxin by sympathetic preganglionic neurones. Neuroscience 40, 805-823.

Chang, H.T., Kuo, H., Whittaker, J.A., Cooper, N.G.F., 1990. Light and electron microscopic analysis of projection neurones retrogradely labeled with Fluoro-Gold: notes on the application of antibodies to Fluoro-Gold. J. Neurosci. Methods 35, 31-37.

Chen, S., Aston-Jones, G., 1998. Axonal collateral-collateral transport of tract tracers in brain neurones: false anterograde labelling and useful tool. Neuroscience 82, 1151-1163.

Connors, B.W., Benardo, L.S., Prince, D.A., 1984. Carbon dioxide sensitivity of dye-coupling among glia and neurones of the neocortex. J. Neurosci. 4 (5), 1324-1330.

Deller, T., Frotscher, M., Nitsch, R., 1995. Morphological evidence for the sprouting of inhibitory commissural fibers in response to the lesion of excitatory entorhinal input to the rat dentate gyrus. J. Neurosci. 15, 6868-6878.

Fink, R.P., Heimer, L., 1967. Two methods for selective silver impregnation of degenerating axons and their synaptic endings in the central nervous system. Brain Res. 4, 369-374.

Fritzsch, B., Sonntag, R., 1991. Sequential double labelling with different fluorescent dyes coupled to dextran amines as a tool to estimate the accuracy of tracer application and or regeneration. J. Neurosci. Methods 39, 9-17.

Garwicz, M., Apps, R., Trott, J.R., 1996. Micro-organization of olivocerebellar and corticonuclear connections of the paravermal cerebellum in the cat. Eur. J. Neurosci. 8, 2726-2738.

Gerfen, C.R., Sawchenko, P.E., 1984. An anterograde neuroanatomical tracing method that shows the detailed morphology of neurones, their axons and terminals: immunohistochemical localization of an axonally transported plant lectin, Phaseolus vulgaris leucoagglutinin (PHA-L). Brain Res. 290, 219-238.

Gibson, A.R., Hansma, D.I., Houk, J.C., Robinson, F.R., 1984. A sensitive low artifact TMB procedure for the demonstration of WGA-HRP in the CNS. Brain Res. 298, 235-241.

Giloh, H., Sedat, J.W., 1982. Fluorescence microscopy: reduced photobleaching of rhodamine and fluorescein protein conjugates by $n$-propyl gallate. Science $217,1252-1255$.

Glover, J.C., Petursdottir, G., Jansen, J.K.S., 1986. Fluorescent dex- tran-amines used as axonal tracers in the nervous system of chicken embryo. J. Neurosci. Methods 18, 243-254.

Godement, P., Vanselow, J., Thanos, S., Bonhoeffer, F., 1987. A study in developing visual systems with a new method of staining neurones and their processes in fixed tissue. Development 101, $697-713$.

Gonatas, N.K., Harper, C., Mizutani, T., Gonatas, J.O., 1979. Superior sensitivity of conjugates of horseradish peroxidase with wheat germ agglutinin for studies of retrograde axonal transport. J. Histochem. Cytochem. 27, 728-734.

Grinvald, A., Salzberg, B.M., Lev-Ram, V., Hildesheim, R., 1987. Optical recording of synaptic potentials from processes of single neurones using intracellular potentiometric dyes. Biophys. J. 51, 643-651.

Hafeli, U.O., Sweeney, S.M., Beresford, B.A., Humm, J.L., Macklis, R.M., 1995. Effective targeting of magnetic radioactive 90Ymicrospheres to tumor cells by an externally applied magnetic field. Preliminary in vitro and in vivo results. Nucl. Med. Biol. 22, 147-155.

Harsch, G.S.J.A., Madison, R.D., 1991. Double-labelling of saphenous nerve neuron pools: a model for determining the accuracy of axon regeneration at the single neuron level. J. Neurosci. Methods 39, 123-130.

Hausmann, R.E., Moscona, A.A., 1976. Isolation of retina-specific cell-aggregating factor from membranes of embryonic neural retina tissue. Proc. Natl. Acad. Sci. USA 73 (10), 3594-3598.

Hong, Y.-M., Thanos, S., 1996. A quantitative approach to identify and isolate pure populations of fluorescently labeled adult retinal ganglion cells using a pressure-driven microaspiration technique. Neurosci. Lett. 214, 111-114.

Honig, M.G., Hume, R.I., 1989. DiI and DiO: versatile fluorescent dyes for neuronal labelling and pathway tracing. TINS 12, 333341 .

Horikawa, K., Armstrong, W.E., 1988. A versatile means of intracellular labelling: injection of biocytin and its detection with avidin conjugates. J. Neurosci. Methods 25, 1-11.

Hubel, D.H., Wiesel, T.N., Stryker, M.P., 1977. Orientation columns in macaque monkey visual cortex demonstrated by the 2-deoxyglucose autoradiographic technique. Nature 269, 328-330.

Janssen, P., Naskar, R., Moore, S., Thanos, S., 1997. Evidence for glaucoma-induced horizontal cell alterations in the human retina. Ger. J. Ophthalmol. 5, 378-385.

Kaneko, T., Saeki, K., Lee, T., Mizuno, N., 1996. Improved retrograde axonal transport and subsequent visualization of tetramethylrhodamine (TMR)-dextran amine by means of an acid injection vehicle and antibodies against TMR. J. Neurosci. Methods 65, 157-165.

Katz, L.C., Iarovici, D.M., 1990. Green fluorescent latex microspheres: a new retrograde tracer. Neuroscience 34, 511-520.

Katz, L.C., Burkhalter, A., Dreyer, W.J., 1984. Fluorescent latex microspheres as a retrograde neuronal marker for in vivo and in vitro studies of visual cortex. Nature 310, 498-500.

Köbbert, C., Thanos, S., 2000. Topographic presentation of the sciatic nerve motoneurons in the spinal cord of the adult rat correlates to region-specific activation patterns of microglia. J. Neurocytol., submitted.

King, V., Armstrong, D.M., Apps, R., Trott, J.R., 1998. Numerical aspects of pontine, lateral reticular, and inferior olivary projections to two paravermal cortical zones of the cat cerebellum. J. Comp. Neurol. 390, 1-15.

Kita, H., Armstrong, W.E., 1991. A biotin-containing compound $N$ (2-aminoethyl) biotinamide for intracellular labeling and neurotracing studies. J. Neurosci. Methods 37, 141-150.

Kristensson, K., 1970. Transport of fluorescent protein tracer in peripheral nerves. Acta Neuropathol. 16, 293-300.

Kristensson, K., Olsson, Y., 1971. Retrograde axonal transport of protein. Brain Res. 29, 363-365. 
Krug, K., Smith, A.L., Thompson, I.D., 1998. The development of topography in the hamster geniculo-cortical projection. J. Neurosci. 18, 5766-5776.

Kuypers, H.G.J.M., Huisman, A.M., 1984. Fluorescent neuronal tracers. Adv. Cell. Neurobiol. 5, 307-340.

Lanciego, J.L., Luquin, M.R., Guillén, J., Giménez-Amaya, J.M., 1998a. Multiple neuroanatomical tracing in primates. Brain Res. Prot. 2 (4), 323-332.

Lanciego, J.L., Goede, P.H., Witter, M.P., Wouterlood, F.G., 1997. Use of peroxidase substrate Vector ${ }^{\circledR}$ VIP for multiple staining in light microscopy. J. Neurosci. Methods 74, 1-7.

Lanciego, J.L., Wouterlood, F.G., Erro, E., Giménez-Amaya, J.M., 1998b. Multiple axonal tracing: simultaneous detection of three tracers in the same section. J. Histochem. Cell Biol. 110 (5), 509515.

Lapper, S.R., Bolam, J.P., 1991. The anterograde and retrograde transport of neurobiotin in the central nervous system of the rat: comparison with biocytin. J. Neurosci. Methods 39, 163-174.

LaVail, J.H., LaVail, M.M., 1972. Retrograde axonal transport in the central nervous system. Science 176, 1416-1417.

Luksch, H., Walkowiak, W., Munoz, A., tenDonkelaar, H.J., 1996. The use of in vitro preparations of the isolated amphibian central nervous system in neuroanatomy and electrophysiology. J. Neurosci. Methods 70, 91-102.

Luksch, H., Cox, K., Karten, H.J., 1998. Bottlebrush dendritic endings and large dendritic fields: motion-detecting neurones in the tectofugal pathway. J. Comp. Neurol. 396 (3), 399-414.

Madison, R., Macklis, J.D., Thies, C., 1990. Latex nanosphere delivery system (LNDS): novel nanometer-sized carries of fluorescent dyes and active agents selectively target neuronal subpopulations via uptake and retrograde transport. Brain Res. 522, 90-98.

Mason, A., Larkman, A., Eldridge, J.L., 1988. A method for intracellular injection of horseradish peroxidase by pressure. J. Neurosci. Methods 22, 181-187.

Mato, M., Ookawara, S., Mato, T.K., Namiki, T., 1985. An attempt to differentiate further between microglia and fluorescent granular perithelial (FGP) cells by their capacity to incorporate exogenous protein. Am. J. Anat. 172, 125-140.

McBride, R.L., Feringa, E.R., Smith, B.E., 1988. The fate of prelabelled Clarke's column neurones after axotomy. Exp. Neurol. 102, 236-243.

Mesulam, M.M., Mufson, E.J., 1980. The rapid anterograde transport of horseradish peroxidase. Neuroscience 5, 1277-1286.

Mesulam, M.M., 1982. Principles of horseradish peroxidase neurochemistry and their applications for tracing neural pathways-axonal transport, enzyme histochemistry and light microscopic analysis. In: Mesulam, M.M. (Ed.), Tracing Neural Connections with Horseradish Peroxidase. IBRO Handbook Series: Methods in the Neurosciences. Wiley, New York, pp. 1-551.

Mey, J., Thanos, S., 1993. Intravitreal injections of neurotrophic factors support the survival of axotomized retinal ganglion cells in adult rats in vivo. Brain Res. 602, 304-317.

Nance, D.M., Burns, J., 1990. Fluorescent dextrans as sensitive anterograde neuroanatomical tracers: applications and pitfalls. Brain Res. Bull. 25, 139-145.

Nauta, W.J.H., Gygax, P.A., 1954. Silver impregnation of degenerating axons in the CNS: a modified technique. Stain Technol. 29, 91-93.

Neuhuber, W., Niederle, B., 1979. Spinal ganglion cells innervating the stomach of rats as demonstrated by somatopetal transport of horseradish peroxidase (HRP). Anat. Embryol. 155, 355-362.

Novikova, L., Novikov, L., Kellerth, J.O., 1997. Persistent neuronal labelling by retrograde fluorescent tracers: a comparison between Fast Blue, Fluoro-Gold and various dextran conjugates. J. Neurosci., Methods 74, 9-15.

Ohm, T.G., Dieckmann, S., 1994. The use of Lucifer Yellow and Mini Ruby for intracellular staining in fixed brain tissue.
Methodological considerations evaluated in rat and human autopsy brains. J. Neurosci. Methods 55, 105-110.

Oscarsson, O., 1980. Functional organization of the olivary projection to the cerebellar anterior lobe. In: Courville, J., De Montingny, C., Lamarre, Y. (Eds.), The Inferior Olivary Nucleus: Anatomy and Physiology. Raven Press, New York, pp. 279-289.

Paxinos, G., Watson, C., 1986. The Rat Brain in Stereotaxic Coordinates, 2nd ed. Academic Press, San Diego.

Rajakumar, N., Elisevich, K., Flumerfelt, B.A., 1993. Biotinylated dextran: a versatile anterograde and retrograde neuronal tracer. Brain Res. 607, 47-53.

Richmond, F.G.R., Gladdy, R., Creasy, J.L., Kitamura, S., Smits, E., Thomson, D.B., 1994. Efficacy of seven retrograde tracers, compared in multiple-labelling studies of feline motoneurons. J. Neurosci. Methods 53, 35-46.

Sawchenko, P.E., Gerfen, C.R., 1985. Plant lectins and bacterial toxins as tools for tracing neuronal connections. Trends Neurosci. 8, 378-385.

Schmid, H., Taylor, D.C., Pierau, F.K., 1983. Tracing of sensory neurones and spinal motoneurons of the pigeon by injection of fluorescent dyes into peripheral nerves. Cell Tissue Res. 232 (1), 9-19.

Schmued, L.C., Fallon, J.H., 1986. Fluoro-Gold: a new fluorescent retrograde axonal tracer with numerous unique properties. Brain Res. 377, 147-154.

Schmued, L.C., Kyriakidis, K., Heimer, L., 1990. In vivo anterograde and retrograde axonal transport of the fluorescent rhodamine-dextran-amine, Fluoro-Ruby, within the CNS. Brain Res. 526, 127-134.

Schmued, L.C., Snavely, L.F., 1993. Photoconversion and electron microscopic localisation of the fluorescent axon tracer FluoroRuby (rhodamine-dextran-amine). J. Histochem. Cytochem. 41, 777-782.

Schofield, B.R., 1990. Uptake of Phaseolus vulgaris leucoagglutinin (PHA-L) by axons of passage. J. Neurosci. Methods 35, 47-56.

Schütte, M., Hoskins, S.G., 1993. Ipsilaterally projecting retinal ganglion cells in Xenopus laevis: an HRP study. J. Comp. Neurol. 331, 482-494.

Senatorov, V.V., Trudeau, V.L., Hu, B., 1997. Immunofluorescence in situ hybridization (IFISH) in neurones retrogradely labelled with rhodamine latex microspheres. Brain Res. Prot. 1, 49-56.

Streit, W.J., Kreutzberg, G.W., 1987. Lectin binding by resting and activated microglia. J. Neurocytol. 16, 249-260.

Streit, W.J., Graeber, M.B., 1993. Heterogeneity of microglia and perivascular cells: insights gained from the facial nucleus paradigm. Glia 7, 68-74.

Ter, Horst, G.J., Groenewegen, H.J., Karst, H., Luiten, P.G.M., 1984. Phaseoulus vulgaris leucoagglutinin immunohistochemistry. A comparison between autoradiographic and lectin tracing of neuronal efferents. Brain Res. 307, 379-383.

Teune, T.M., van der Burg, J., de Zeeuw, C.I., Voogd, J., Ruigrok, T.J.H., 1998. Single Purkinje cell can innervate multiple classes of projection neurones in the cerebellar nuclei of the rat: a light microscopic and ultrastructural triple-tracer study in the rat. J. Comp. Neurol. 392, 164-178.

Thanos, S., Bonhoeffer, F., 1983. Investigations on development and topographic order of retinotectal axons: anterograde and retrograde staining of axons and their perikarya with rhodamine in vivo. J. Comp. Neurol. 219, 420-430.

Thanos, S., Bonhoeffer, F., 1987. Axonal arborization in the developing chick retinotectal system. J. Comp. Neurol. 261, 155-164.

Thanos, S., Rohrbach, J.M., Thiel, H.J., 1991. Post mortem preservation of ganglion cells in the human retina: a morphometric investigation with the carbocyanine dye DiI. Retina 11, 318-327.

Thanos, S., Mey, J., 1992. Ganglion cells in the juvenile chick retina 
and their ability to regenerate axons in vitro. Exp. Eye Res. 54, 377-391.

Thanos, S., Seeger, J., Kazca, J., Mey, J., 1994. Old dyes for new scopes: the phagocytosis-dependent long-term fluorescence labelling of microglial cells in vivo. Trends Neurosci. 17, 177-182.

Thanos, S., Mey, J., 1995. Type-specific stabilization and targetdependent survival of regenerating ganglion cells in the retina of adult rats. J. Neurosci. 15, 1057-1079.

Trott, J.R., Apps, R., Armstrong, D.M., 1990. Topographical organisations within the cerebellar nucleocortical projection to the cerebellar cortex of lobule $\mathrm{Vb} / \mathrm{c}$ in the cat. Exp. Brain Res. 80, 415428.

Trott, J.R., Apps, R., 1993. Zonal organisation within the projection from the inferior olive to the rostral paramedian lobule of the cat cerebellum. Eur. J. Neurosci. 5, 162-173.

Trott, J.R., Apps, R., Armstrong, D.M., 1998a. Zonal organisation of cortico-nuclear and nucleo-cortical projections of the paramedian lobule of the cat cerebellum. Part 1: The C1 zone. Exp. Brain Res. 118, 298-315.

Trott, J.R., Apps, R., Armstrong, D.M., 1998b. Zonal organisation of cortico-nuclear and nucleo-cortical projections of the paramedian lobule of the cat cerebellum. Part 2: The C2 zone. Exp. Brain Res. 118, 316-330.

Trowe, T., Klosterman, S., Baier, H., Granato, M., Crawford, A.D., Grunewald, B., Hoffmann, H., Karlstrom, R.O., Meyer, S.U., Müller, B., Richter, S., Nüsslein-Volhard, C., Bonhoeffer, F., 1996. Mutations disrupting the ordering and topographic mapping of axons in the retinotectal projection of the zebrafish, Dario rerio. Development 123, 439-450.

Van, Bockstaele, E.J., Wright, A.M., Cestari, D.M., Pickel, V.M., 1994. Immunolabeling of retrogradely transported fluorogold. Sensitivity and application of ultrastructural analysis of transmitter-specific mesolimbic circuitry. J. Neurosci. Methods 55, 65-78.

Van, der Want, J.J.L., Klooster, J., Nunes, Cardoso, B., de, Weerd, H., Liem, R.S.B., 1997. Tract-tracing in the nervous system of vertebrates using horseradish peroxidase and its conjugates: tracers, chromogens and stabilization for light and electron microscopy. Brain Res. Prot. 1, 269-279.

Vanselow, J., Thanos, S., Godement, P., Henke-Fahle, S., Bonhoeffer, F., 1989. Spatial arrangement of radial glia and ingrowing of retinal axons in the chick optic tectum during development. Dev. Brain Res. 45, 15-27.
Veenman, C.L., Reiner, A., Honig, M.G., 1992. Biotinylated dextran amine as an anterograde tracer for single- and double-labeling studies. J. Neurosci. Methods 41, 239-254.

Vince, G.H., Bouterfa, H., Goldbrunner, R., Roosen, K., Tonn, J.C., 1997. Fast blue, a fluorescent tracer in glioma cell culture, affects cell proliferation and motility. Neurosci. Lett. 233, 148150.

Voogd, J., Ruigrok, T.J.H., 1997. Transverse and longitudinal patterns in the mammalian cerebellum. In: De Zeeuw, C.I., Strata, P., Voogd, J. (Eds.), The cerebellum: From Structure to Control. Prog. Brain Res. 114; 21-37.

Waggoner, A., 1976. Optical probes of membrane potential. J. Membr. Biol. 27, 317-324.

Weiss, P., Hiscoe, H.N., 1948. Experiments on the mechanism of nerve growth. J. Exp. Zool. 107, 315-395.

Wessendorf, M.W., 1991. Fluoro-Gold: composition, and mechanism of uptake. Brain Res. 553, 135-148.

Wirsig-Wiechmann, C.R., 1994. Biocytin: a neuronal tracer compatible with rapid decalcification procedures. J. Neurosci. Methods $51,213-216$.

Witter, M.P., 1993. Organization of the entorhinal-hippocampal system: a review of current anatomical data. Hippocampus 3, 33-44.

Wouterlood, F.G., Jorritsma-Byham, B., Goede, P.H., 1990a. Combination of anterograde tracing with Phaseolus vulgaris-leucoagglutinin, retrograde fluorescent tracing and fixed-slice intracellular injection of Lucifer yellow. J. Neurosci. Methods 33, 207-217.

Wouterlood, F.G., Goede, P.H., Groenewegen, H.J., 1990b. The in situ detectability of the neuroanatomical tracer Phaseolus vulgaris-leucoagglutinin (PHA-L). J. Comp. Neuroanatom. 3, 11-18.

Wouterlood, F.G., Jorritsma-Byham, B., 1994. The anterograde neuroanatomical tracer biotinylated dextran amine: comparison with the tracer PHA-L in preparations for electron microscopy. J. Neurosci. Methods 48, 75-87.

Zecevic, D., Antic, S., 1998. Fast optical measurement of membrane potential changes at multiple sites on an individual nerve cell. J. Histochem. 30, 197-216.

Zhou, M., Grofova, I., 1995. The use of peroxidase substrate Vector $^{\circledR}$ VIP in electron microscopic single and double antigen localization. J. Neurosci. Methods 62, 149-158. 